FIAN/TD-16/96

ITEP/TH-47/96

\title{
Non-perturbative Quantum Theories and Integrable Equations
}

\author{
A.Marshakov \\ Theory Department, P. N. Lebedev Physics Institute, Leninsky prospect, 53, Moscow, 117924, Russia \\ and ITEP, Moscow 117259, Russia
}

\begin{abstract}
I review the appearance of classical integrable systems as an effective tool for the description of nonperturbative exact results in quantum string and gauge theories. Various aspects of this relation: spectral curves, action-angle variables, Whitham deformations and associativity equations are considered separately demonstrating hidden parallels between topological $2 d$ string theories and naively non-topological $4 d$ theories. The proofs are supplemented by explicit illustrative examples.
\end{abstract}

${ }^{1}$ E-mail address: mars@lpi.ac.ru 


\section{Introduction}

In this paper I will try to describe recent results in modern quantum field theory and string theory related to the exact construction of non-perturbative solutions and their formulation in terms of integrable systems. Starting from quantum field theory the problem is to compute exactly the quantum spectrum and the correlation functions

$$
\left\langle\Phi_{k_{1}} \ldots \Phi_{k_{n}}\right\rangle=\int D \varphi e^{-\frac{1}{\hbar} \int \mathcal{L}\left(\varphi, \partial_{\mu} \varphi\right)} \Phi_{\mathbf{k}_{1}}\left(\varphi, \partial_{\mu} \varphi, \ldots\right) \ldots \Phi_{\mathbf{k}_{n}}\left(\varphi, \partial_{\mu} \varphi, \ldots\right) \equiv F_{\mathbf{k}_{1}, \ldots, \mathbf{k}_{n}}(t, u)
$$

as functions of coupling constants $\left\{t_{k}\right\}$, quantum characteristics of physical operators $\left\{\mathbf{k}_{1}, \ldots, \mathbf{k}_{n}\right\}$ and possible parameters of classical solutions $\left\{u_{\alpha}\right\}$ or moduli of the theory.

Unfortunately almost never the integral in (1) can be computed exactly. Up to now the only positive experience of computing (11) comes from perturbative calculations and/or lattice regularization of (11) resulting usually in some approximations being quite far from the exact answer. In string theory the situation is even more complicated since there is no real target-space formulation of the problem: the Polyakov path integral

$$
\mathcal{F}(\Lambda)=\sum_{\text {genus }} \Lambda^{g} F_{g} \quad F_{g}=\int_{\Sigma_{g}} D g D x e^{-\frac{1}{\hbar} \int \mathcal{L}(x, g)}
$$

is by definition only a perturbative formulation.

Recent investigations showed however (see for example [1, 2, 3, 4, 5, 6, 6, 8, 9]) that sometimes there exists a way to find explicitly the exact nonperturbative results (spectrum, correlation functions, effective actions) even in the quantum theories which can not be considered as quantum integrable models (see for example [10, 11] and references therein) at least in conventional and naive sense. In contrast to "naive" quantum integrable models where usually it is (infinite-dimensional) algebra of quantum symmetry which allows one to calculate the spectrum and the correlation functions, there is no even to such extent "straightforward" way in the theories I am going to discuss. The intention to study these particular models is caused by hope that they are not so far away from the realistic quantum gauge and string theories where the solution to the basic problems of confinement and quantum gravity is looked for, being on the other hand solvable at least in the sense to be discussed below.

The starting point is that even in this class of models there are still no direct ways of solution - like there are no such ways for in many other models of quantum field and string theory. Amazingly enough, the indirect arguments show that all existing examples of the exact non-perturbative solutions have almost identically the same effective formulation which can be presented in terms of effective completely integrable model forgetting about many properties of the bare theory 1 . The low-energy limit of such models is also described by a sort of topological theory - which is not topological in naive sense still having lots of properties of conventional (essentially $2 d$ ) topological models.

It is still not possible to describe the mechanism which obtains the final results starting directly from the Lagrangian formulation ?, however in what follows I will try to believe that it exists and formulate the basic

\footnotetext{
${ }^{1}$ In particular there is no real distinction between the theories living in different space-time dimensions.

${ }^{2}$ One hope is that the arising equations of hydrodynamical type might be considered as generalizations of the renormalization-group technique of the perturbation theory. Indeed it is known that in the perturbation theory the scale dependence of quantum
} 
features of the effective description, which attract a lot of interest from the point of view of integrable systems themselves.

First, the parameters of the theory - or its moduli come - usually from the (gauge-invariant) low-energy values of the (background) fields in the target-space. For example, in $4 d$ supersymmetric gauge theories these are in part given by the v.e.v.'s of the Higgs fields $h_{k}=\frac{1}{k}\left\langle\operatorname{Tr} \Phi^{k}\right\rangle$, in string theories - by the moduli of the target-space metric (e.g. Kähler or complex structures), gauge fields (e.g. moduli of flat connections or selfdual gauge fields) etc. The problem itself is to find the (exact nonperturbative) dependence of the physical objects upon the moduli parameters 3 . The idea that the moduli of (some) target-space theories can be identified with the moduli of the complex manifolds (in particular having the property that their different values can be treated as equivalent if related by an action of some (discrete) group) is usually called as duality concept (see for example [12]) while the action of group itself - a duality map.

A crucial point is that the class of the theories we discuss is distinguished by the holomorphic dependence of the (complex) moduli parameters - i.e. there exists a complex structure on the moduli space and only functions with "good" global behaviour enter the game. This goes back to the holomorphic structures arising in the instantonic calculus and to the Belavin-Knizhnik theorem in string theory leading to drastical simplifications removing the possible ambiguity in the form of the exact answer. In the known examples the moduli of the theory appear to be (a subspace in) the moduli space of complex structures of the target-space spectral curves $\Sigma$ 7. It turns out, that nonperturbatively the target-space spectral curve acquires a nontrivial topological structure (being just defined locally - as a sphere of the scale parameter in perturbation theory) and complex structure on the spectral curve is parameterized by moduli of the theory. This additional complicated structure means that "stringy" nature plays an important role in the nonperturbative formulation and strings (and $D$-branes) wrapping along topologically nontrivial directions produce important effects in the exact effective formulation 13 while perturbatively the spectral curve can be tested only "locally".

To be precise, the relation between the non-perturbative solutions and integrable theories was described in detail for some topological $2 d$ theories and so far for the Seiberg-Witten (SW) solutions only for particular families of models: the most known is the $\mathcal{N}=2 \mathrm{SYM}$ theory with one $\left(N_{a}=1\right)$ "matter" $\mathcal{N}=2$ hypermultiplet in the adjoint representation of the gauge group $G$ - which is known to be related to the Calogero-Moser family correlation functions is related with their dependence on the coupling constants by means of the first order equation

$$
\left(\frac{d}{d \log \mu}-\sum \beta_{i}(g) \frac{\partial}{\partial g_{i}}\right) F(g ; \mu)=0
$$

Naively in the exact solution there is no scale dependence, so the resulting equation could be of the form $\sum \beta_{i}(g) \frac{\partial F}{\partial g_{i}}=0$, or the derivatives over $\mu$ could be replaced by the derivatives over moduli $\left\{u_{\alpha}\right\}$. Even accepting this it is necessary to stress that there is no known way to define $\beta$-function beyond the perturbation theory (i.e. not using the scale dependence $\left.\beta_{i}(g)=\frac{\partial g_{i}}{\partial \log \mu}\right)$. However, one should also remember that some of the integrable equations - for example the associativity equations discussed in the last section look like having a purely string theory nature.

${ }^{3}$ Of course, in addition to the parametric dependence of moduli themselves the physical quantities can depend on the topological (discrete) characteristics of moduli spaces, moreover in the simplest topological string models only this dependence is essential and the correlation functions can be just numbers.

${ }^{4}$ From the general properties of string theories one can expect that the same picture exists when the moduli of the theory are effectively described as moduli of higher dimensional complex manifolds (K3, the Calabi-Yau 3-folds etc.). The particular case considered in this text corresponds to a specific situation when the CY manifold effectively degenerates into $1_{\mathbf{C}} d$ complex curve $\Sigma$ 13. 
of integrable systems 14, 15, 16, 17, 18. When the hypermultiplet decouples (its mass becomes infinite), the dimensional transmutation takes place and the pure gauge $4 d \mathcal{N}=2 \mathrm{SYM}$ theory gets associated with the Toda-chain model [9]. It is also known [19, 20] that the $N_{c}=3, N_{f}=2$ curve can be associated with the Goryachev-Chaplygin top while a natural suggestion for the whole $\mathcal{N}=2$ SQCD family is to associate it with the well-known family of integrable systems - (in general inhomogeneous) $s l(2)$ spin chains, with the Toda chain (pure gauge model) being again a limiting case. The crucial motivation for such a suggestion 19] is the specific quadratic form of the spectral equations, derived in 422 . 2 .

Now, once the solutions are formulated in terms of periods of some differentials on a complex spectral curve an integrable system (moreover the particular class of systems of KP/Toda type - where the Liouville torus is restricted to be a real section of Jacobian of a complex curve) arises more or less by definition due to the Krichever construction (see 24] and references therein). This very useful observation leads to a possibility of applying rather simple technique of Lax pairs, spectral curves, symplectic forms and $\tau$-functions to naively infinite-dimensional quantum field and string theories. Again it means that the effective non-perturbative theory can be formulated in the language of (finite-dimensional) integrable systems, i.e. only small part of all degrees of freedom is essential for the exact non-perturbative behaviour.

The plan of this paper looks as follows. First, I consider the fact of appearance of curves and integrable systems and formulate the string/QFT results in these terms. Next, in sect.3 it is shown that a completely integrable system arises after one introduces [24] a meromorphic 1-form $d S$ whose derivatives

$$
\frac{\partial d S}{\partial h_{k}} \cong d \omega_{k}
$$

give holomorphic differentials and it is explained that the generating 1-form defines a completely integrable system on a symplectic manifold endowed with symplectic form $\Omega \sim \delta d S$ which in all cases we discuss can be essentially written as

$$
d S=\lambda d \log w=\operatorname{Tr} \mathcal{L} d \log T \quad \Omega=\delta \lambda \wedge \delta \log w=\operatorname{Tr} \delta \mathcal{L} \wedge \delta \log T
$$

The symplectic form (4) is defined by the eigenvalues of two operators. Quasiclassically their common spectrum defines the spectral curve. The symplectomorphisms of (4) can be considered as transformations between the dual integrable systems with the generating function $S=\sum_{k} \int^{\gamma_{k}} d S$.

Indeed, writing (函) more explicitly one finds that $\Omega=\left.\sum_{k} \delta \lambda \wedge \delta \log w\right|_{\gamma_{k}}=\sum_{k} \delta h_{k} \wedge \delta \phi_{k}$ and the Hamiltonian flows provided by $h_{k}$ produce a completely integrable system on Jacobian with co-ordinates $\left\{\phi_{k}\right\}$. Next, in sect.4 another property of the same 1-differential $d S$ is discussed - $d S$ (4) plays the role of the generating differential of the Whitham hierarchy describing the flows in moduli space around a point, corresponding to a finite-gap solution. The basic features of the Whitham hierarchy are presented and some explicit examples of its solutions are considered. Finally in sect.5 the central object containing the most complete information about the integrable system $\log \mathcal{T}=\log \mathcal{T}_{0}+\log \mathcal{T}_{\theta}$ is discussed. Its part (a logariphm of a quasiclassical $\tau$ - function) restricted to the dependence on moduli

$$
\left.\log \mathcal{T}_{0}\right|_{\text {moduli }} \equiv \mathcal{F}
$$

\footnotetext{
${ }^{5}$ Another possibility [25] is to stay within the frames of the Toda chain modifying only the boundary conditions (i.e. changing $T$ operator for the same $\mathcal{L}$ ). This however seems to be not enough to consider the situation with $N_{f}>N_{c}$.
} 
(usually called a prepotential) is defined in terms of the "periods" $t_{k}=\oint_{C_{k}} d S$ or $t_{\alpha}=\operatorname{res}_{P_{\alpha}}\left(\lambda^{-\alpha} d S\right)$ and intersection form $T_{i k}=\int_{\Sigma} d \omega_{i} \wedge d \omega_{j}$ on $\Sigma$. The main statement of sect.5 is that the prepotential $\mathcal{F}=\log \mathcal{T}_{0}$ in general satisfies the associativity equation having the form (for the matrices $\mathcal{F}_{i j k}=\frac{\partial^{3} \mathcal{F}}{\partial t_{i} \partial t_{j} \partial t_{k}} \equiv\left(\mathcal{F}_{i}\right)_{j k}$ )

$$
\mathcal{F}_{i} \mathcal{F}_{j}^{-1} \mathcal{F}_{k}=\mathcal{F}_{k} \mathcal{F}_{j}^{-1} \mathcal{F}_{i} \quad \forall i, j, k
$$

In Conclusion some open questions are listed and the relation with string dualities is discussed.

\section{Curves and Integrable systems}

The simplest example of the nonperturbative solutions is when they exist in explicit form, being related to the rational spectral curve $\Sigma$ where only the times connected to the residues are valid, the prepotential $\mathcal{F}=\frac{t_{1}^{3}}{6}+\ldots$ is polynomial in times and the eigenvalues of two operators in (何) are polynomial functions on Riemann sphere $C P^{1}$. The topological correlators are numbers and count the intersection indices on moduli space - this is an example of topological gravity [27, 3, 歫.

The case of physical ( $c<1$ or $p q-)$ gravity is known much less explicitly and correspond already to nontrivial spectral curves $\Sigma_{g=\frac{(p-1)(q-1)}{2}}$ 228] Formally the topological $2 d$ theories with the "target-space" higher genus spectral curves were constructed in [29, 30].

The higher genus complex curves arise also in $4 d$ SUSY gauge theories - the field theory limit $\alpha^{\prime} \rightarrow 0$ of $c>1$ supersymmetric string theories

$$
\mathcal{L}=\int d^{4} \theta F\left(\Phi_{i}\right)=\ldots \frac{1}{g^{2}} \operatorname{Tr} F_{\mu \nu}^{2}+i \theta \operatorname{Tr} F_{\mu \nu} \tilde{F}_{\mu \nu}+\ldots
$$

(the superfield $\Phi_{i}=\varphi^{i}+\theta \sigma_{\mu \nu} \tilde{\theta} G_{\mu \nu}^{i}+\ldots$ ) where the nonperturbative exact solution is formally defined as a map

$$
G, \tau, h_{k} \rightarrow a_{i}, a_{i}^{D}, a_{i}^{D}=\frac{\partial \mathcal{F}}{\partial a_{i}} \quad T_{i j}=\frac{\partial^{2} \mathcal{F}}{\partial a_{i} \partial a_{j}} \quad M \sim\left|\mathbf{n a}+\mathbf{m a}^{D}\right|
$$

$G$ is gauge group, $\tau$ - the UV coupling constant, $h_{k}=\frac{1}{k}\left\langle\operatorname{Tr} \Phi^{k}\right\rangle$ - the v.e.v.'s of the Higgs field) and an elegant description in terms of $\Sigma_{g=\operatorname{rank} G}$ with $h_{k}$ parameterizing some of the (in most cases hyperelliptic) moduli of complex structures. The periods of meromorphic 1-form (ब) $a_{i}=\oint_{A_{i}} d S, a_{i}^{D}=\oint_{B_{i}} d S$ determine the BPS massive spectrum, $a_{i}^{D}=\frac{\partial \mathcal{F}}{\partial a^{i}}$ the prepotential $\mathcal{F}$ (giving the low-energy effective action) and, thus, the set of low-energy coupling constants $T_{i j}=\frac{\partial^{2} \mathcal{F}}{\partial a_{i} \partial a_{j}}=\frac{\partial a_{i}^{D}}{\partial a_{j}}$. The curves $\Sigma_{g=\operatorname{rank} G}$ are special spectral curves $\square$ of the nontrivial finite-gap solutions to the periodic Toda-chain problem and its natural deformations.

\subsection{Toda chain: $N_{c} \times N_{c}$ versus $2 \times 2$ representation}

We start our analysis from the simplest Toda-chain model, which in the framework of the nonperturbative solutions corresponds to the $4 d$ pure gauge $\mathcal{N}=2$ supersymmetric Yang-Mills theory 21, 9. The periodic problem in this model can be formulated in two different ways, which will be further deformed into two different

\footnotetext{
${ }^{6}$ This is however the case where the exact form of the duality transformation - relating the partition functions in the dual points - is known exactly, having the form of a Fourier transform with the exponent $S=\int^{\lambda} d S$.

${ }^{7}$ They correspond to the $g$-parametric families of complex curves in the moduli space of total dimension $3 g-3$.
} 
directions. These deformations are hypothetically related to the two different couplings of the $4 d$ theory by adding the adjoint and fundamental matter $\mathcal{N}=2$ hypermultiplets correspondingly.

The Toda chain system can be defined by the equations of motion

$$
\frac{\partial q_{i}}{\partial t}=p_{i} \quad \frac{\partial p_{i}}{\partial t}=e^{q_{i+1}-q_{i}}-e^{q_{i}-q_{i-1}}
$$

where one assumes (for the periodic problem with the "period" $N_{c}$ ) that $q_{i+N_{c}}=q_{i}$ and $p_{i+N_{c}}=p_{i}$. It is an integrable system, with $N_{c}$ Poisson-commuting Hamiltonians, $h_{1}^{T C}=\sum p_{i}, h_{2}^{T C}=\sum\left(\frac{1}{2} p_{i}^{2}+e^{q_{i}-q_{i-1}}\right)$, etc. As any finite-gap solution the periodic problem in Toda chain is described in terms of (the eigenvalues and the eigenfunctions of) two operators: the Lax operator $\mathcal{L}$ (or the auxiliary linear problem for (9))

$$
\lambda \psi_{n}^{ \pm}=\sum_{k} \mathcal{L}_{n k} \psi_{k}^{ \pm}=e^{\frac{1}{2}\left(q_{n+1}-q_{n}\right)} \psi_{n+1}^{ \pm}+p_{n} \psi_{n}^{ \pm}+e^{\frac{1}{2}\left(q_{n}-q_{n-1}\right)} \psi_{n-1}^{ \pm}\left(= \pm \frac{\partial}{\partial t} \psi_{n}^{ \pm}\right)
$$

and the second is a monodromy or shift operator in a discrete variable - the number of a particle

$$
T q_{n}=q_{n+N_{c}} \quad T p_{n}=p_{n+N_{c}} \quad T \psi_{n}=\psi_{n+N_{c}}
$$

The common spectrum of these two operators

$$
\mathcal{L} \psi=\lambda \psi \quad T \psi=w \psi \quad[\mathcal{L}, T]=0
$$

means that there exists a relation between them $\mathcal{P}(\mathcal{L}, T)=0$ which can be strictly formulated in terms of spectral curve $\Sigma: \mathcal{P}(\lambda, w)=0$. The generation function for these Hamiltonians can be written in terms of $\mathcal{L}$ and $T$ operators and the Toda chain possesses two essentially different formulations of this kind.

In the first version (which can be considered as a limiting case of Hitchin system [31]), the Lax operator (10) is written in the basis of the $T$-operator eigenfunctions and becomes the $N_{c} \times N_{c}$ matrix,

$$
\mathcal{L}^{T C}(w)=\left(\begin{array}{ccccc}
p_{1} & e^{\frac{1}{2}\left(q_{1}-q_{2}\right)} & 0 & & w e^{\frac{1}{2}\left(q_{1}-q_{N_{c}}\right)} \\
e^{\frac{1}{2}\left(q_{2}-q_{1}\right)} & p_{2} & e^{\frac{1}{2}\left(q_{2}-q_{3}\right)} & \ldots & 0 \\
0 & e^{\frac{1}{2}\left(q_{3}-q_{2}\right)} & p_{3} & 0 \\
\frac{1}{w} e^{\frac{1}{2}\left(q_{N_{c}}-q_{1}\right)} & 0 & 0 & \\
& & \ldots & \\
& & & p_{N_{c}}
\end{array}\right)
$$

defined on the two-punctured sphere. The Poisson brackets $\left\{p_{i}, q_{j}\right\}=\delta_{i j}$ imply that it satisfies a PB relation

$$
\left\{\mathcal{L}^{T C}(w) \stackrel{\otimes}{,} \mathcal{L}^{T C}\left(w^{\prime}\right)\right\}=\left[\mathcal{R}\left(w, w^{\prime}\right), \mathcal{L}^{T C}(w) \otimes \mathbf{1}+\mathbf{1} \otimes \mathcal{L}^{T C}\left(w^{\prime}\right)\right]
$$

with the numeric trigonometric $\mathcal{R}$-matrix,

$$
\mathcal{R}\left(w, w^{\prime}\right)=\frac{w \sum\left(\delta_{i, i+1} \otimes \delta_{i+1, i}\right)+\left(w^{\prime} \sum \delta_{i+1, i} \otimes \delta_{i, i+1}\right)}{w-w^{\prime}}
$$

and the eigenvalues of the Lax operator defined from the spectral equation

$$
\mathcal{P}(\lambda, w)=\operatorname{det}_{N_{c} \times N_{c}}\left(\mathcal{L}^{T C}(w)-\lambda\right)=0
$$


are Poisson-commuting with each other. Substituting the explicit expression (13) into (16), one gets [32]:

$$
w+\frac{1}{w}=2 P_{N_{c}}(\lambda)
$$

or

$$
y^{2}=P_{N_{c}}^{2}(\lambda)-1 \quad 2 y=w-\frac{1}{w}
$$

where $P_{N_{c}}(\lambda)$ is a polynomial of degree $N_{c}$, with the coefficients being the Schur polynomials of the Hamiltonians $h_{k}=\sum_{i=1}^{N_{c}} p_{i}^{k}+\ldots$ :

$$
P_{N_{c}}(\lambda)=\left(\lambda^{N_{c}}+h_{1} \lambda^{N_{c}-1}+\frac{1}{2}\left(h_{2}-h_{1}^{2}\right) \lambda^{N_{c}-2}+\ldots\right)
$$

The spectral equation depends only on the mutually Poisson-commuting combinations of the dynamical variables - the Hamiltonians or better action variables - parameterizing (a subspace in the) moduli space of the complex structures of the hyperelliptic curves $\Sigma^{T C}$ of genus $N_{c}-1=\operatorname{rank} S U\left(N_{c}\right)$.

An alternative description of the same system arises when one solves explicitly the auxiliary linear problem (10) which is a second-order difference equation - to solve which one just rewrites it as $\tilde{\psi}_{i+1}=L_{i}^{T C}(\lambda) \tilde{\psi}_{i}$ (after a simple "gauge" transformation) with the help of a chain of $2 \times 2$ Lax matrices [10]

$$
L_{i}^{T C}(\lambda)=\left(\begin{array}{cc}
p_{i}+\lambda & e^{q_{i}} \\
e^{-q_{i}} & 0
\end{array}\right), \quad i=1, \ldots, N_{c}
$$

These matrices obey the quadratic $r$-matrix Poisson relations [33]

$$
\left\{L_{i}^{T C}(\lambda) \stackrel{\otimes}{,} L_{j}^{T C}\left(\lambda^{\prime}\right)\right\}=\delta_{i j}\left[r\left(\lambda-\lambda^{\prime}\right), L_{i}^{T C}(\lambda) \otimes L_{j}^{T C}\left(\lambda^{\prime}\right)\right]
$$

with the ( $i$-independent!) numerical rational $r$-matrix satisfying the classical Yang-Baxter equation $r(\lambda)=$ $\frac{1}{\lambda} \sum_{a=1}^{3} \sigma_{a} \otimes \sigma^{a}$. As a consequence, the transfer matrix (generally defined for the inhomogeneous lattice with inhomogenities $\lambda_{i}$ 's)

$$
T_{N_{c}}(\lambda)=\prod_{1 \geq i \geq N_{c}}^{\curvearrowleft} L_{i}\left(\lambda-\lambda_{i}\right)
$$

satisfies the same Poisson-bracket relation

$$
\left\{T_{N_{c}}(\lambda) \stackrel{\otimes}{,} T_{N_{c}}\left(\lambda^{\prime}\right)\right\}=\left[r\left(\lambda-\lambda^{\prime}\right), T_{N_{c}}(\lambda) \otimes T_{N_{c}}\left(\lambda^{\prime}\right)\right]
$$

and the integrals of motion of the Toda chain are generated by another form of spectral equation

$$
\operatorname{det}_{2 \times 2}\left(T_{N_{c}}^{T C}(\lambda)-w\right)=w^{2}-w \operatorname{Tr} T_{N_{c}}^{T C}(\lambda)+\operatorname{det} T_{N_{c}}^{T C}(\lambda)=w^{2}-w \operatorname{Tr} T_{N_{c}}^{T C}(\lambda)+1=0
$$

or

$$
\mathcal{P}(\lambda, w)=\operatorname{Tr} T_{N_{c}}^{T C}(\lambda)-w-\frac{1}{w}=2 P_{N_{c}}(\lambda)-w-\frac{1}{w}=0
$$

(We used the fact that $\operatorname{det}_{2 \times 2} L^{T C}(\lambda)=1$ leads to $\operatorname{det}_{2 \times 2} T_{N_{c}}^{T C}(\lambda)=1$.) The r.h.s. of (25) is a polynomial of degree $N_{c}$ in $\lambda$, with the coefficients being the integrals of motion since

$$
\begin{gathered}
\left\{\operatorname{Tr} T_{N_{c}}(\lambda), \operatorname{Tr} T_{N_{c}}\left(\lambda^{\prime}\right)\right\}=\operatorname{Tr}\left\{T_{N_{c}}(\lambda) \stackrel{\otimes}{,} T_{N_{c}}\left(\lambda^{\prime}\right)\right\}= \\
=\operatorname{Tr}\left[r\left(\lambda-\lambda^{\prime}\right), T_{N_{c}}(\lambda) \otimes T_{N_{c}}\left(\lambda^{\prime}\right)\right]=0
\end{gathered}
$$


For the particular choice of $L$-matrix (20), the inhomogenities of the chain, $\lambda_{i}$, can be absorbed into the redefinition of the momenta $p_{i} \rightarrow p_{i}-\lambda_{i}$.

In what follows we consider possible elliptic deformations of two Lax representations of the Toda chain. The deformation of the $N_{c} \times N_{c}$ representation provides the Calogero-Moser model while the deformation of the spin-chain $2 \times 2$ representation gives rise to the Sklyanin $X Y Z$ model.

In addition to the curve (16), (17), (18) and (25) to define the Toda-chain system one needs a generating 1-differential $d S^{T C}$. I will discuss it in detail later now only mentioning that $d S^{T C}=\lambda \frac{d w}{w}$ in the Toda chain case has exactly the form of (4) where $\lambda$ in this example is a hyperelliptic co-ordinate in (17) and (18). Its periods

$$
\mathbf{a}=\oint_{\mathbf{A}} d S^{T C}=\oint_{\mathbf{A}} \lambda \frac{d w}{w} \quad \mathbf{a}_{D}=\oint_{\mathbf{B}} d S^{T C}=\oint_{\mathbf{B}} \lambda \frac{d w}{w}
$$

define the BPS massive spectrum and prepotential of the pure $\mathcal{N}=2$ SUSY Yang-Mills theory.

\subsection{Elliptic deformation of the $N_{c} \times N_{c}$ representation: the Calogero-Moser model}

The $N_{c} \times N_{c}$ matrix Lax operator for the $G L\left(N_{c}\right)$ Calogero system is 34]

$$
\begin{gathered}
\mathcal{L}^{C a l}(\xi)=\left(\mathbf{p H}+\sum_{\boldsymbol{\alpha}} F(\mathfrak{q} \boldsymbol{\alpha} \mid \xi) E_{\boldsymbol{\alpha}}\right)= \\
=\left(\begin{array}{cccc}
p_{1} & F\left(\mathfrak{q}_{1}-\mathfrak{q}_{2} \mid \xi\right) & \ldots & F\left(\mathfrak{q}_{1}-\mathfrak{q}_{N_{c}} \mid \xi\right) \\
F\left(\mathfrak{q}_{2}-\mathfrak{q}_{1} \mid \xi\right) & p_{2} & \ldots & F\left(\mathfrak{q}_{2}-\mathfrak{q}_{N_{c}} \mid \xi\right) \\
& & \ldots & \\
& & & \\
F\left(\mathfrak{q}_{N_{c}}-\mathfrak{q}_{1} \mid \xi\right) & F\left(\mathfrak{q}_{N_{c}}-\mathfrak{q}_{2} \mid \xi\right) & \ldots & p_{N_{c}}
\end{array}\right)
\end{gathered}
$$

The function $F(\mathfrak{q} \mid \xi)=\frac{g}{\omega} \frac{\sigma(\mathfrak{q}+\xi)}{\sigma(\mathfrak{q}) \sigma(\xi)} e^{\zeta(\mathfrak{q}) \xi}$ is expressed in terms of the Weierstrass elliptic functions and, thus, the Lax operator $\mathcal{L}(\xi)$ is defined on the elliptic curve $E(\tau)$ (complex torus with periods $\omega, \omega^{\prime}$ and modulus $\tau=\frac{\omega^{\prime}}{\omega}$ ). The Calogero coupling constant is $\frac{g^{2}}{\omega^{2}} \sim m^{2}$, where in the $4 d$ interpretation $m$ plays the role of the mass of the adjoint matter $\mathcal{N}=2$ hypermultiplet breaking $\mathcal{N}=4$ SUSY down to $\mathcal{N}=2$ [14].

The spectral curve $\Sigma^{C a l}$ for the $G L\left(N_{c}\right)$ Calogero system is given by:

$$
\operatorname{det}_{N_{c} \times N_{c}}\left(\mathcal{L}^{C a l}(\xi)-\lambda\right)=0
$$

The BPS masses $\mathbf{a}$ and $\mathbf{a}_{D}$ are now the periods of the generating 1-differential

$$
d S^{C a l} \cong \lambda d \xi
$$

along the non-contractable contours on $\Sigma^{C a l}$. Integrability of the Calogero system is implied by the Poisson structure of the form

$$
\left\{\mathcal{L}(\xi) \stackrel{\otimes}{,} \mathcal{L}\left(\xi^{\prime}\right)\right\}=\left[\mathcal{R}_{12}^{C a l}\left(\xi, \xi^{\prime}\right), \mathcal{L}(\xi) \otimes \mathbf{1}\right]-\left[\mathcal{R}_{21}^{C a l}\left(\xi, \xi^{\prime}\right), \mathbf{1} \otimes \mathcal{L}\left(\xi^{\prime}\right)\right]
$$

with the dynamical elliptic $\mathcal{R}$-matrix [35], guaranteeing that the eigenvalues of the matrix $\mathcal{L}$ are in involution. 
In order to recover the Toda-chain system, one takes the double-scaling limit [36], when $g \sim m$ and $-i \tau$ both go to infinity (and $\mathfrak{q}_{i}-\mathfrak{q}_{j}=\frac{1}{2}\left[(i-j) \log g+\left(q_{i}-q_{j}\right)\right]$ ) so that the dimensionless coupling $\tau$ gets substituted by a dimensional parameter $\Lambda^{N_{c}} \sim m^{N_{c}} e^{i \pi \tau}$. In this limit, the elliptic curve $E(\tau)$ degenerates into the (twopunctured) Riemann sphere with coordinate $w=e^{\xi} e^{i \pi \tau}$ so that

$$
d S^{C a l} \rightarrow d S^{T C} \cong \lambda \frac{d w}{w}
$$

The Lax operator of the Calogero system turns into that of the $N_{c}$-periodic Toda chain (13):

$$
\mathcal{L}^{C a l}(\xi) d \xi \rightarrow \mathcal{L}^{T C}(w) \frac{d w}{w}
$$

and the spectral curve acquires the form of (16). In contrast to the Toda case, 29) can not be rewritten in the form (17) and specific $w$-dependence of the spectral equation (16) is not preserved by embedding of Toda into Calogero-Moser particle system. However, the form $(\sqrt{17})$ can be naturally preserved by the alternative deformation of the Toda-chain system when it is considered as (a particular case of) a spin-chain model.

To deal with the "elliptic" deformations of the Toda chain below, we will use a non-standard normalization of the Weierstrass $\wp$-function defined by

$$
\wp(\xi \mid \tau)=\sum_{m, n=-\infty}^{+\infty} \frac{1}{(\xi+m+n \tau)^{2}}-\sum_{m, n=-\infty}^{+\infty} \frac{1}{(m+n \tau)^{2}}
$$

so that it is double periodic in $\xi$ with periods 1 and $\tau=\frac{\omega^{\prime}}{\omega}$ (that differs from a standard definition by a factor of $\omega^{-2}$ and by rescaling $\left.\xi \rightarrow \omega \xi\right)$. According to $(34)$, the values of $\wp(\xi \mid \tau)$ in the half-periods, $e_{a}=e_{a}(\tau), a=1,2,3$, are also the functions only of $\tau$ - again differing by a factor of $\omega^{-2}$ from the conventional definition. The complex torus $E(\tau)$ can be defined as $\mathbf{C} / \mathbf{Z} \oplus \tau \mathbf{Z}$ with a "flat" co-ordinate $\xi$ defined modulo(1, $\tau)$. Alternatively, any torus (with a marked point) can be described as elliptic curve

$$
y^{2}=\left(x-e_{1}\right)\left(x-e_{2}\right)\left(x-e_{3}\right) \quad x=\wp(\xi) \quad y=-\frac{1}{2} \wp^{\prime}(\xi)
$$

and the canonical holomorphic 1-differential is

$$
d \xi=2 \frac{d x}{y}
$$

In the simplest example of $N_{c}=2$, the spectral curve $\Sigma^{C a l}$ has genus 2. Indeed, in this particular case, eq.(29) turns into

$$
\mathcal{P}(\lambda ; x, y)=\lambda^{2}-h_{2}+\frac{g^{2}}{\omega^{2}} x=0
$$

This equation says that with any value of $x$ one associates two points of $\Sigma^{C a l}, \lambda= \pm \sqrt{h_{2}-\frac{g^{2}}{\omega^{2}} x}$, i.e. it describes $\Sigma^{c a l}$ as a double covering of the elliptic curve $E(\tau)$ ramified at the points $x=\left(\frac{\omega}{g}\right)^{2} h_{2}$ and $x=\infty$. In fact, since $x$ is an elliptic coordinate on $E(\tau)$ (when elliptic curve itself is treated as a double covering over the Riemann sphere $\left.C P^{1}\right), x=\left(\frac{\omega}{g}\right)^{2} h_{2}$ corresponds to a pair of points on $E(\tau)$ distinguished by the sign of $y$. This would be true for $x=\infty$ as well, but $x=\infty$ is one of the branch points in our parameterization (35) of $E(\tau)$. Thus, the two cuts between $x=\left(\frac{\omega}{g}\right)^{2} h_{2}$ and $x=\infty$ on every sheet of $E(\tau)$ touching at the common end at $x=\infty$ become effectively a single cut between $\left(\left(\frac{\omega}{g}\right)^{2} h_{2},+\right)$ and $\left(\left(\frac{\omega}{g}\right)^{2} h_{2},-\right)$. Therefore, we can 
consider the spectral curve $\Sigma^{C a l}$ as two tori $E(\tau)$ glued along one cut, i.e. $\Sigma_{N_{c}=2}^{C a l}$ has genus 2 . It turns out to be a hyperelliptic curve (for $N_{c}=2$ only!) after substituting in (37) $x$ from the second equation to the first one.

Two holomorphic 1-differentials on $\Sigma^{C a l}$ can be chosen to be

$$
v=\frac{d x}{y} \sim \frac{\lambda d \lambda}{y} \quad V=\frac{d x}{y \lambda} \sim \frac{d \lambda}{y}
$$

so that

$$
d S \cong \lambda d \xi=\sqrt{h_{2}-\frac{g^{2}}{\omega^{2}} \wp(\xi)} d \xi=\frac{d x}{y} \sqrt{h_{2}-\frac{g^{2}}{\omega^{2}} x}
$$

It is easy to check the basic property (3):

$$
\frac{\partial d S}{\partial h_{2}} \cong \frac{1}{2} \frac{d x}{y \lambda}
$$

The fact that only one of two holomorphic 1-differentials (38) appears at the r.h.s. is related to their different parity with respect to the $\mathbf{Z}_{2} \otimes \mathbf{Z}_{2}$ symmetry of $\Sigma^{C a l}: y \rightarrow-y$ and $\lambda \rightarrow-\lambda$. Since $d S$ has certain parity, its integrals along the two of four elementary non-contractable cycles on $\Sigma^{C a l}$ automatically vanish leaving only two non-vanishing quantities $a$ and $a_{D}$, as necessary for the $4 d$ interpretation. Moreover, these two non-vanishing integrals can be actually evaluated in terms of the "reduced" genus-one curve

$$
Y^{2}=(y \lambda)^{2}=\left(h_{2}-\frac{g^{2}}{\omega^{2}} x\right) \prod_{a=1}^{3}\left(x-e_{a}\right),
$$

with $d S \cong\left(h_{2}-\frac{g^{2}}{\omega^{2}} x\right) \frac{d x}{Y}$. Since now $x=\infty$ is no longer a ramification point, $d S$ obviously has simple poles at $x=\infty$ (at two points on the two sheets of $\Sigma_{\text {reduced }}^{\text {Cal }}$ ) with the residues $\pm \frac{g}{\omega} \sim \pm m$.

The opposite limit of the Calogero-Moser system with vanishing coupling constant $g^{2} \sim m^{2} \rightarrow 0$ corresponds to the $\mathcal{N}=4$ SUSY Yang-Mills theory with identically vanishing $\beta$-function. The corresponding integrable system is a collection of free particles and the generating differential $d S \cong \sqrt{h_{2}} \cdot d \xi$ is just a holomorphic differential on $E(\tau)$.

\subsection{From Toda to Spin Chains}

Now, let us discuss another deformation of the Toda chain corresponding to the coupling of the $\mathcal{N}=2 \mathrm{SYM}$ theory to the fundamental matter. According to [7], [22], the spectral curves for the $\mathcal{N}=2$ SQCD with any $N_{f}<2 N_{c}$ have the same form as (12) with a less trivial monodromy matrix

$$
\operatorname{Tr} T_{N_{c}}(\lambda)=P_{N_{c}}(\lambda)+R_{N_{c}-1}(\lambda), \quad \operatorname{det} T_{N_{c}}(\lambda)=Q_{N_{f}}(\lambda),
$$

and $Q_{N_{f}}(\lambda)$ and $R_{N_{c}-1}(\lambda)$ are certain $h$-independent polynomials of $\lambda$ (remind that for the Toda chain with the Lax matrix (20) $\operatorname{det}_{2 \times 2} T_{N_{c}}^{\mathrm{TC}}(\lambda)=\prod_{i=1}^{N_{c}} \operatorname{det}_{2 \times 2} L_{i}^{\mathrm{TC}}\left(\lambda-\lambda_{i}\right)=1$ and $\left.\operatorname{Tr} T_{N_{c}}^{\mathrm{TC}}(\lambda)=P_{N_{c}}(\lambda)\right)$. Two descriptions, (16) and (24), are identically equivalent for the Toda chain, but their deformations are very different: the "chain" representation (24), (25) is naturally embedded into the family of $X Y Z$ spin chains [10, 33].

Our proposal is to look at the orthogonal to the previous generalization of the Toda chain, i.e. deform eqs.(21)-(26) preserving the Poisson brackets

$$
\begin{gathered}
\left\{L(\lambda) \stackrel{\otimes}{,} L\left(\lambda^{\prime}\right)\right\}=\left[r\left(\lambda-\lambda^{\prime}\right), L(\lambda) \otimes L\left(\lambda^{\prime}\right)\right], \\
\left\{T_{N_{c}}(\lambda) \stackrel{\otimes}{,} T_{N_{c}}\left(\lambda^{\prime}\right)\right\}=\left[r\left(\lambda-\lambda^{\prime}\right), T_{N_{c}}(\lambda) \otimes T_{N_{c}}\left(\lambda^{\prime}\right)\right],
\end{gathered}
$$


and, thus, the possibility to build a monodromy matrix $T(\lambda)$ by multiplication of $L_{i}(\lambda)$ 's. The full spectral curve for the periodic inhomogeneous spin chain is given by:

$$
\operatorname{det}\left(T_{N_{c}}(\lambda)-w\right)=0,
$$

with the inhomogeneous $T$-matrix still defined by (22), satisfying (43), while generating 1 -form is now

$$
d S=\lambda \frac{d W}{W}
$$

In the case of $s l(2)$ spin chains, the spectral equation can be written as

$$
w+\frac{\operatorname{det}_{2 \times 2} T_{N_{c}}(\lambda)}{w}=\operatorname{Tr}_{2 \times 2} T_{N_{c}}(\lambda),
$$

or

$$
W+\frac{1}{W}=\frac{\operatorname{Tr}_{2 \times 2} T_{N_{c}}(\lambda)}{\sqrt{\operatorname{det}_{2 \times 2} T_{N_{c}}(\lambda)}} .
$$

The r.h.s. of this equation contains the dynamical variables of the spin system only in the special combinations - its Hamiltonians (which are all in involution, i.e. Poisson-commuting). It is this peculiar shape (quadratic $w$ dependence) that suggests the identification of the periodic $\operatorname{sl}(2)$ spin chains with solutions to the SW problem with the fundamental matter supermultiplets.

\section{4 $X X X$ Spin Chain and the Low Energy SYM with $N_{f}<2 N_{c}$}

The $2 \times 2$ Lax matrix for the $\operatorname{sl}(2) X X X$ chain is

$$
L(\lambda)=\lambda \cdot \mathbf{1}+\sum_{a=1}^{3} S_{a} \cdot \sigma^{a}
$$

The Poisson brackets of the dynamical variables $S_{a}, a=1,2,3$ (taking values in the algebra of functions) are implied by (43) with the rational $r$-matrix

$$
r(\lambda)=\frac{1}{\lambda} \sum_{a=1}^{3} \sigma^{a} \otimes \sigma^{a} .
$$

In the $\operatorname{sl}(2)$ case, they are just

$$
\left\{S_{a}, S_{b}\right\}=i \epsilon_{a b c} S_{c},
$$

i.e. $\left\{S_{a}\right\}$ plays the role of angular momentum ("classical spin") giving the name "spin-chains" to the whole class of systems. Algebra (50) has an obvious Casimir operator (an invariant, Poisson-commuting with all the generators $S_{a}$ ),

$$
K^{2}=\mathbf{S}^{2}=\sum_{a=1}^{3} S_{a} S_{a},
$$

so that

$$
\begin{gathered}
\operatorname{det}_{2 \times 2} L(\lambda)=\lambda^{2}-K^{2}, \\
\operatorname{det}_{2 \times 2} T_{N_{c}}(\lambda)=\prod_{i=N_{c}}^{1} \operatorname{det}_{2 \times 2} L_{i}\left(\lambda-\lambda_{i}\right)=\prod_{i=N_{c}}^{1}\left(\left(\lambda-\lambda_{i}\right)^{2}-K_{i}^{2}\right)= \\
=\prod_{i=N_{c}}^{1}\left(\lambda+m_{i}^{+}\right)\left(\lambda+m_{i}^{-}\right)=Q_{2 N_{c}}(\lambda),
\end{gathered}
$$


where we assumed that the values of spin $K$ can be different at different nodes of the chain, and

$$
m_{i}^{ \pm}=-\lambda_{i} \mp K_{i}
$$

While the determinant of monodromy matrix (52) depends on dynamical variables only through the Casimir

values $K_{i}$ of the Poisson algebra, the dependence of the trace $\mathcal{T}_{N_{c}}(\lambda)=\frac{1}{2} \operatorname{Tr}_{2 \times 2} T_{N_{c}}(\lambda)$ is less trivial. Still, as usual for integrable systems, it depends on $S_{a}^{(i)}$ only through the Hamiltonians of the spin chain (which are not Casimirs but Poisson-commute with each other).

In order to get some impression how the Hamiltonians look like, we present explicit examples of monodromy matrices for $N_{c}=2$ and 3. Hamiltonians depend non-trivially on the $\lambda_{i}$-parameters (inhomogeneities of the chain) and the coefficients in the spectral equation (44) depend only on the Hamiltonians and symmetric functions of the $m$-parameters (53), i.e. the dependence of $\left\{\lambda_{i}\right\}$ and $\left\{K_{i}\right\}$ is rather special. This property is crucial for identification of the $m$-parameters with the masses of the matter supermultiplets in the $\mathcal{N}=2$ SQCD. The explicit examples can be found in [23].

\section{5 $\mathrm{N}_{f}=2 \mathrm{~N}_{c}$ : Generic spin-chain models and Sklyanin algebra}

However, the story can not be complete without studying the most intriguing "elliptic" case of $N_{f}=2 N_{c}$, when the $4 d$ theory is supposed to be UV-finite (at least at some particular values of moduli) and then possesses an extra dimensionless parameter: the UV non-abelian coupling constant $\tau=\frac{8 \pi i}{e^{2}}+\frac{\theta}{\pi}$.

The most general theory of this sort is known as Sklyanin $X Y Z$ spin chain with the elementary $L$-operator defined on the elliptic curve $E(\tau)$ and is explicitly given by (see [10] and references therein):

$$
L^{S k l}(\xi)=S^{0} \mathbf{1}+i \frac{g}{\omega} \sum_{a=1}^{3} W_{a}(\xi) S^{a} \sigma_{a}
$$

where

$$
\begin{gathered}
W_{a}(\xi)=\sqrt{e_{a}-\wp(\xi \mid \tau)}=i \frac{\theta_{11}^{\prime}(0) \theta_{a+1}(\xi)}{\theta_{a+1}(0) \theta_{11}(\xi)} \\
\theta_{2} \equiv \theta_{01}, \quad \theta_{3} \equiv \theta_{00}, \quad \theta_{4} \equiv \theta_{10}
\end{gathered}
$$

Let us note that our spectral parameter $\xi$ is connected with the standard one $u$ [10 by the relation $u=2 K \xi$, where $K \equiv \int_{0}^{\frac{\pi}{2}} \frac{d t}{\sqrt{1-k^{2} \sin ^{2} t}}=\frac{\pi}{2} \theta_{00}^{2}(0), k^{2} \equiv \frac{e_{1}-e_{2}}{e_{1}-e_{3}}$ so that $K \rightarrow \frac{\pi}{2}$ as $q \rightarrow 0$. This factor results into additional multiplier $\pi$ in the trigonometric functions in the limiting cases below.

The Lax operator (54) satisfies the Poisson relation (21) with the numerical elliptic $r$-matrix $r(\xi)=$ $i \frac{g}{\omega} \sum_{a=1}^{3} W_{a}(\xi) \sigma_{a} \otimes \sigma_{a}$, which implies that $S^{0}, S^{a}$ form the (classical) Sklyanin algebra [39, 33]:

$$
\begin{gathered}
\left\{S^{a}, S^{0}\right\}=2 i\left(\frac{g}{\omega}\right)^{2}\left(e_{b}-e_{c}\right) S^{b} S^{c} \\
\left\{S^{a}, S^{b}\right\}=2 i S^{0} S^{c}
\end{gathered}
$$

with the obvious notation: $a b c$ is the triple 123 or its cyclic permutations.

\footnotetext{
${ }^{8}$ Eq. (53) implies that the limit of vanishing masses, all $m_{i}^{ \pm}=0$, is associated with the homogeneous chain (all $\lambda_{i}=0$ ) and vanishing spins at each site (all $K_{i}=0$ ). It deserves noting that a similar situation was considered by L.Lipatov 37 in the study of the high-energy limit of the ordinary (non-supersymmetric) QCD. The spectral equation is then the classical limit of the Baxter equation from [38].
} 
The coupling constant $\frac{g}{\omega}$ can be eliminated by simultaneous rescaling of the $S$-variables and the symplectic form:

$$
S^{a}=\frac{\omega}{g} \hat{S}^{a} \quad S^{0}=\hat{S}^{0} \quad\{,\} \rightarrow \frac{g}{\omega}\{,\}
$$

Then

$$
\begin{gathered}
L(\xi)=\hat{S}^{0} \mathbf{1}+i \sum_{a=1}^{3} W_{a}(\xi) \hat{S}^{a} \sigma_{a} \\
\left\{\hat{S}^{a}, \hat{S}^{0}\right\}=2 i\left(e_{b}-e_{c}\right) \hat{S}^{b} \hat{S}^{c} \quad\left\{\hat{S}^{a}, \hat{S}^{b}\right\}=2 i \hat{S}^{0} \hat{S}^{c}
\end{gathered}
$$

In the rational limit both $\omega, \omega^{\prime} \rightarrow \infty$, then (56) turns into

$$
\begin{gathered}
\left\{S^{a}, S^{0}\right\}=0 \\
\left\{S^{a}, S^{b}\right\}=2 i \epsilon^{a b c} S^{0} S^{c}
\end{gathered}
$$

i.e. $S^{0}$ itself becomes a Casimir operator (constant), while the remaining $S^{a}$ form a classical angular-momentum (spin) vector (50). The corresponding Lax operator (48) $L \equiv \lambda L_{X X X}=\lambda \mathbf{1}+\mathbf{S} \cdot \boldsymbol{\sigma}$ describes the $X X X$ spin chain with the rational $r$-matrix (49).

The determinant $\operatorname{det}_{2 \times 2} \hat{L}(\xi)$ is equal to

$$
\operatorname{det}_{2 \times 2} \hat{L}(\xi)=\hat{S}_{0}^{2}+\sum_{a=1}^{3} e_{a} \hat{S}_{a}^{2}-\wp(\xi) \sum_{a=1}^{3} \hat{S}_{a}^{2}=K-M^{2} \wp(\xi)=K-M^{2} x
$$

where

$$
K=\hat{S}_{0}^{2}+\sum_{a=1}^{3} e_{a}(\tau) \hat{S}_{a}^{2} \quad M^{2}=\sum_{a=1}^{3} \hat{S}_{a}^{2}
$$

are the Casimir operators of the Sklyanin algebra (i.e. Poisson commuting with all the generators $\hat{S}^{0}, \hat{S}^{1}, \hat{S}^{2}$, $\left.\hat{S}^{3}\right)$. The determinant of the monodromy matrix (22) is

$$
Q(\xi)=\operatorname{det}_{2 \times 2} T_{N_{c}}(\xi)=\prod_{i=1}^{N_{c}} \operatorname{det}_{2 \times 2} \hat{L}\left(\xi-\xi_{i}\right)=\prod_{i=1}^{N_{c}}\left(K_{i}-M_{i}^{2} \wp\left(\xi-\xi_{i}\right)\right)
$$

while the trace $P(\xi)=\frac{1}{2} \operatorname{Tr} T_{N_{c}}(\xi)$ generates mutually Poisson-commuting Hamiltonians, since

$$
\left\{\operatorname{Tr} T_{N_{c}}(\xi), \operatorname{Tr} T_{N_{c}}\left(\xi^{\prime}\right)\right\}=0
$$

For example, in the case of the homogeneous chain (all $\xi_{i}=0$ in (63)) $\operatorname{Tr} T_{N_{c}}(\xi)$ is a combination of the polynomials:

$$
P(\xi)=\operatorname{Pol}_{\left[\frac{N_{c}}{2}\right]}^{(1)}(x)+y \operatorname{Pol}_{\left[\frac{N_{c}-3}{2}\right]}^{(2)}(x),
$$

where $\left[\frac{N_{c}}{2}\right]$ is integral part of $\frac{N_{c}}{2}$, and the coefficients of $\operatorname{Pol}^{(1)}$ and $\mathrm{Pol}^{(2)}$ are Hamiltonians of the $X Y Z$ model 9. As a result, the spectral equation (47) for the $X Y Z$ model acquires the form:

$$
w+\frac{Q(\xi)}{w}=2 P(\xi)
$$

\footnotetext{
${ }^{9}$ For the inhomogeneous chain the explicit expression for the trace is more sophisticated: one should make use of the formulas like

$$
\wp\left(\xi-\xi_{i}\right)=\left(\frac{\wp^{\prime}(\xi)+\wp^{\prime}\left(\xi_{i}\right)}{\wp(\xi)-\wp\left(\xi_{i}\right)}\right)^{2}-\wp(\xi)-\wp\left(\xi_{i}\right)=4\left(\frac{y+y_{i}}{x-x_{i}}\right)^{2}-x-x_{i}
$$
}


where for the homogeneous chain $P$ and $Q$ are polynomials in $x=\wp(\xi)$ and $y=\frac{1}{2} \wp^{\prime}(\xi)$. Eq. (66) describes the double covering of the elliptic curve $E(\tau)$ : with generic point $\xi \in E(\tau)$ one associates the two points of $\Sigma^{X Y Z}$, labeled by two roots $w_{ \pm}$of equation $(66)$. The ramification points correspond to $w_{+}=w_{-}= \pm \sqrt{Q}$, or $Y=\frac{1}{2}\left(w-\frac{Q}{w}\right)=\sqrt{P^{2}-Q}=0$.

The curve (66) is in fact similar to that of $N_{c}=2$ Calogero-Moser system (37). The difference is that now $x=\infty$ is not a branch point, therefore, the number of cuts on the both copies of $E(\tau)$ is $N_{c}$ and the genus of the spectral curve is $N_{c}+1$. Rewriting analytically $\Sigma^{X Y Z}$ as a system of equations

$$
\begin{gathered}
y^{2}=\prod_{a=1}^{3}\left(x-e_{a}\right), \\
Y^{2}=P^{2}-Q
\end{gathered}
$$

the set of holomorphic 1-differentials on $\Sigma^{X Y Z}$ can be chosen as

$$
v=\frac{d x}{y}, \quad V_{\alpha}=\frac{x^{\alpha} d x}{y Y} \quad \alpha=0, \ldots,\left[\frac{N_{c}}{2}\right] \quad \tilde{V}_{\beta}=\frac{x^{\beta} d x}{Y} \quad \beta=0, \ldots,\left[\frac{N_{c}-3}{2}\right]
$$

with the total number of holomorphic 1-differentials $1+\left(\left[\frac{N_{c}}{2}\right]+1\right)+\left(\left[\frac{N_{c}-3}{2}\right]+1\right)=N_{c}+1$ being equal to the genus of $\Sigma^{X Y Z}$.

Now, given the spectral curve one can try to write down the "generating" 1-differential $d S$ which obeys the basic defining property (3). For the Toda chain it can be chosen in two different ways

$$
d \Sigma^{T C} \cong d \lambda \log w \quad d S^{T C} \cong \lambda \frac{d w}{w} \quad d \Sigma^{T C}=-d S^{T C}+d f^{T C}
$$

Both $d \Sigma^{T C}$ and $d S^{T C}$ obey the basic property (3) and, while $f^{T C}$ itself is not its variation, $\delta f^{T C}=\lambda \frac{\delta w}{w}$ appears to be a (meromorphic) single-valued function on $\Sigma^{T C}$. In the $X X X$ case [23], one has almost the same formulas as $(69)$

$$
d \Sigma^{X X X} \cong d \lambda \log W \quad d S^{X X X} \cong \lambda \frac{d W}{W} \quad d \Sigma^{X X X}=-d S^{X X X}+d f^{X X X} \quad W \equiv \frac{w}{\sqrt{\operatorname{det} T_{N_{c}}(\lambda)}}
$$

For the $X Y Z$ model (66) the generating 1-form(s) $d S^{X Y Z}$ can be defined as

$$
d \Sigma^{X Y Z} \cong d \xi \cdot \log W \quad d S^{X Y Z} \cong \xi \frac{d W}{W}=-d \Sigma^{X Y Z}+d(\xi \log W)
$$

Now, under the variation of moduli (which are all contained in $P$, while $Q$ is moduli independent),

$$
\delta\left(d \Sigma^{X Y Z}\right) \cong \frac{\delta W}{W} d \xi=\frac{\delta P(\xi)}{\sqrt{P(\xi)^{2}-Q(\xi)}} d \xi=\frac{d x}{y Y} \delta P
$$

and, according to (10), the r.h.s. is a holomorphic 1-differential on the spectral curve (66). The singularities of $d \Sigma^{X Y Z}$ are located at the points where $W=0$ or $W=\infty$, i.e. at zeroes of $Q(\xi)$ or poles of $P(\xi)$. In the vicinity of a singular point, $d \Sigma^{X Y Z}$ is not single-valued but acquires addition $2 \pi i d \xi$ when circling around this point. The difference between $d \Sigma$ and $d S$ is again a total derivative, but $\delta f^{X Y Z}=\xi \frac{\delta W}{W}$ is not a single-valued function. In contrast to $d \Sigma^{X Y Z}, d S^{X Y Z}$ has simple poles at $W=0, \infty$ with the residues $\left.\xi\right|_{w=0, \infty}$, which are defined modulo $1, \tau$. Moreover, $d S^{X Y Z}$ itself is multivalued: it changes by $(1, \tau) \times \frac{d W}{W}$ when circling along non-contractable cycles on $E(\tau)$.

Naively, neither $d \Sigma^{X Y Z}$ nor $d S^{X Y Z}$ can naively play the role of the Seiberg-Witten 1-form - which is believed to possess well-defined residues, interpreted as masses of the matter hypermultiplets [7]. 
One can naturally assume that the $X Y Z$ chain, which is an elliptic deformation of the $X X X$ chain known to describe $\mathcal{N}=2$ supersymmetric QCD with $N_{f}<2 N_{c}$, can be associated with the $N_{f}=2 N_{c}$ case. This would provide a description of the conformal (UV-finite) supersymmetric QCD, differing from the conventional one [7. 22]. However, as demonstrated above, there are several serious differences between $X Y Z$ and $X X X$ models, which should be kept in mind.

1) Normally, there are two natural ways to introduce generating 1-form - these are represented by the meromorphic 1-differentials $d S$ and $d \Sigma$ in the main text. Usually, both satisfy (3); $d \Sigma$ has no simple poles, but is not single-valued on $\Sigma$, while $d S$ is single-valued and possesses simple poles (of course, in general $d S \not d \Sigma$ ). The proper generating 1-form is $d S$. However, in the $X Y Z$ case, both $d S$ and $d \Sigma$ are not single-valued. Moreover, the residues of $d S$ - identified with masses of the matter hypermultiplets in the framework of [7] - are defined only modulo $(1, \tau)$.

2) As $\tau \rightarrow i \infty$, the $X Y Z$ model turns into $X X Z$ rather than $X X X$ one. This makes the description of the "dimensional transmutation" regime rather tricky.

3) Starting from the spectral curve (16) for the Toda-chain (pure $\mathcal{N}=2 \mathrm{SYM}$ ), the Calogero-Moser deformation is associated with "elliptization" of the $w$-variable, while the $X Y Z$-deformation - with that of the $\lambda$-variable. It is again nontrivial to reformulate the theory in such a way that the both deformations become of the same nature. One of the most naive pictures would associate the Hitchin-type (Calogero) models with the "insertion" of an $S L\left(N_{c}\right)$-orbit at one puncture on the elliptic curve, while the spin-chain $(X Y Z)$ models - with the $S L(2)$-orbits at $N_{c}$ punctures. Alternatively, one can say that the $X Y Z$-type "elliptization", while looking local (i.e. $L$-operators at every site are deformed independently), is in fact a global one (all the $L$ 's can be elliptized only simultaneously, with the same $r$-matrix and $\tau$ ), - but this is not clearly reflected in existing formalism, discussed in this section. Moreover, the proper formalism should naturally allow one to include any simple Lie group (not only $S L\left(N_{c}\right)$ ) and any representations (not only adjoint or fundamental).

Already these comments are enough to demonstrate that the hottest issues of integrability and quantum group theory (like notions of elliptic groups and dynamical $R$-matrices) can be of immediate importance for the Seiberg-Witten (and generic duality) theory.

\section{$3 \quad$ Symplectic structure}

Now let us turn to the discussion of a more subtle point - why the generating 1-form (14) indeed describes an integrable system. To do this I will discuss the symplectic structure on the space of the finite-gap solutions. This symplectic structure was introduced in 24] and recently proposed in 25] as coming directly from the symplectic form on the space of all the solutions to the hierarchy. Below, a very simple and straightforward proof of this result is presented [26] and the relation with the analogous object in low-dimensional non-perturbative string theory [40] is discussed.

To prove that (四) is a generating one-form of the whole hierarchy one starts with the variation of the generating function

$$
S(\Sigma, \gamma)=\sum_{i} \int^{\gamma_{i}} E d p
$$


(where $d E$ and $d p\left(=d \lambda\right.$ and $\left.=\frac{d w}{w}\right)$ in the particular case above) are two meromorphic differentials on a spectral curve $\Sigma$ and $\gamma$ is the divisor of the solution (poles of the BA function))

$$
\delta S=\sum_{i}(E d p)\left(\gamma_{i}\right)+\sum_{i} \int^{\gamma_{i}} \delta E d p \quad \delta^{2} S=\delta\left(\sum_{i}(E d p)\left(\gamma_{i}\right)\right)+\sum_{i}(\delta E d p)\left(\gamma_{i}\right)
$$

From $\delta^{2} S=0$ it follows that

$$
\varpi=\delta E \wedge \delta p=\delta\left(\sum_{i}(E d p)\left(\gamma_{i}\right)\right)=-\sum_{i}(\delta E d p)\left(\gamma_{i}\right)
$$

Now, the variation $\delta E$ (for constant $p$ ) follows from the Lax equation (auxiliary linear problem)

$$
\frac{\partial}{\partial t} \psi=\mathcal{L} \psi(=E \psi)
$$

so that

$$
\delta E=\frac{\left\langle\psi^{\dagger} \delta \mathcal{L} \psi\right\rangle}{\left\langle\psi^{\dagger} \psi\right\rangle}
$$

and one concludes that

$$
\varpi=-\left\langle\delta \mathcal{L} \sum_{i}\left(d p \frac{\psi^{\dagger} \psi}{\left\langle\psi^{\dagger} \psi\right\rangle}\right)\left(\gamma_{i}\right)\right\rangle
$$

Let us turn to several important examples.

$\mathbf{K P} / \mathbf{K d V}$. In the KP-case the equation (76) looks as

$$
\frac{\partial}{\partial t} \psi=\left(\frac{\partial^{2}}{\partial x^{2}}+u\right) \psi(=E \psi)
$$

therefore the equation (78) implied by $\left\langle\psi^{\dagger} \psi\right\rangle=\int_{d x} \psi^{\dagger}(x, P) \psi(x, P)$ and $\delta \mathcal{L}=\delta u(x)$ gives

$$
\varpi=-\int_{d x} \delta u(x) \sum_{i}\left(\frac{d p}{\left\langle\psi^{\dagger} \psi\right\rangle} \psi^{\dagger}(x) \psi(x)\right)\left(\gamma_{i}\right)
$$

The differential $d \Omega=\frac{d p}{\left\langle\psi^{\dagger} \psi\right\rangle} \psi^{\dagger}(x) \psi(x)$ is holomorphic on $\Sigma$ except for the "infinity" point $P_{0}$ where it has zero residue 41]. Its variation 10

$$
\tilde{\delta}\left(\operatorname{res}_{P_{0}} d \Omega+\sum_{i} \operatorname{res}_{\gamma_{i}} d \Omega\right)=0
$$

can be rewritten as

$$
\delta v(x)+\sum_{i} d \Omega\left(\gamma_{i}\right)=0
$$

where $v(x)$ is a "residue" of the BA function at the point $P_{0}$ obeying $v^{\prime}(x)=u(x)$. Substituting (82) into (78) one gets

$$
\varpi=\int_{d x} \delta u(x) \int_{d x^{\prime}}^{x} \delta u\left(x^{\prime}\right)
$$

or the first symplectic structure of the KdV equation.

Toda chain/lattice. (The case directly related to the pure SYM theory). One has

$$
\left\langle\psi^{\dagger} \psi\right\rangle=\sum_{n} \psi_{n}^{+}(P) \psi_{n}^{-}(P)
$$

and the Lax equation acquires the form (10) where $t=t_{+}+t_{-}$and $t_{1}=t_{+}-t_{-}$is the first time of the Toda chain, so that

$$
\delta \lambda=\frac{\sum_{k} \psi_{k}^{+} \delta p_{k} \psi_{k}^{-}}{\left\langle\psi^{+} \psi^{-}\right\rangle}
$$

\footnotetext{
${ }^{10}$ It should be pointed out that the variation $\tilde{\delta}$ corresponds to a rather specific situation when one shifts only $\psi$ keeping $\psi^{\dagger}$ fixed.
} 
and $(78)$ becomes

$$
\varpi=-\sum_{k} \delta p_{k} \sum_{i}\left(\frac{d p}{\left\langle\psi^{+} \psi^{-}\right\rangle} \psi_{k}^{+} \psi_{k}^{-}\right)\left(\gamma_{i}\right)
$$

and to get

$$
\varpi=\sum_{k} \delta p_{k} \wedge \delta q_{k}
$$

one has to prove

$$
\sum_{i}\left(\frac{d p}{\left\langle\psi^{+} \psi^{-}\right\rangle} \psi_{k}^{+} \psi_{k}^{-}\right)\left(\gamma_{i}\right)=\delta q_{k}
$$

To do this one considers again

$$
\tilde{\delta}\left(\operatorname{res}_{P_{+}}+\operatorname{res}_{P_{-}}+\sum_{i} \operatorname{res}_{\gamma_{i}}\right) d \Omega_{n}=0 \quad d \Omega_{n}=\frac{d p}{\left\langle\psi^{+} \psi^{-}\right\rangle} \psi_{n}^{+} \psi_{n}^{-}
$$

where the first two terms for $\psi_{n}^{ \pm} \underset{\lambda \rightarrow \lambda\left(P_{ \pm}\right)}{\sim} e^{ \pm q_{n}} \lambda^{ \pm n}\left(1+\mathcal{O}\left(\lambda^{-1}\right)\right)$ satisfying two "shifted" equations (10) (with $\tilde{q}_{n}$ and $q_{n}$ correspondingly) give $\delta q_{n}=\tilde{q}_{n}-q_{n}$ while the rest - the l.h.s. of (87).

Calogero-Moser system. Introducing the "standard" $d E$ and $d p$ one the curve $\Sigma$ (29) with the 1-form (30) where $d p=d \xi$ is holomorphic on torus $\oint_{A} d p=\omega, \oint_{B} d p=\omega^{\prime}$ and $E=\lambda$ has $n-1$ poles with residue $=1$ and 1 pole with residue $=-(n-1)$, the BA function is defined by 34

$$
\mathcal{L}^{C a l}(\xi) \mathbf{a}=\lambda \mathbf{a}
$$

with the essential singularities

$$
a_{i} \underset{E=E_{+}}{\sim} e^{x_{i} \zeta(\xi)}(1+\mathcal{O}(\xi)) \quad a_{i} \underset{E \neq E_{+}}{\sim} e^{x_{i} \zeta(\xi)}\left(-\frac{1}{n-1}+\mathcal{O}(\xi)\right)
$$

and (independent of dynamical variables) poles $\gamma$. Hence, similarly to the above case for the eq. (78) one has $\left\langle\psi^{\dagger} \psi\right\rangle=\sum_{i} a_{i}^{\dagger}(P) a_{i}(P), \delta \mathcal{L}^{C a l}=\frac{\sum_{i} a_{i}^{\dagger}(P) \delta p_{i} a_{i}(P)}{\sum_{i} a_{i}^{\dagger}(P) a_{i}(P)}$ so that

$$
\varpi=-\sum_{k} \delta p_{k} \sum_{i}\left(\frac{d \xi}{\left\langle a^{\dagger} a\right\rangle} a_{k}^{\dagger} a_{k}\right)\left(\gamma_{i}\right)
$$

and the residue formula

$$
\tilde{\delta}\left(\sum_{P_{j}: p=0} \operatorname{res}_{P_{j}}+\sum_{i} \operatorname{res}_{\gamma_{i}}\right) d \Omega_{k}=0 \quad d \Omega_{k}=\frac{d \xi}{\left\langle a^{\dagger} a\right\rangle} a_{k}^{\dagger} a_{k}
$$

where the first sum is over all "infinities" $p=0$ at each sheet of the cover (29). After variation and using (90) it gives again

$$
\varpi=\sum_{k} \delta p_{k} \wedge \delta x_{k}
$$

The general proof of the more cumbersome analog of the above derivation can be found in [25]. To show how the above formulas work explicitly, let us, finally, demonstrate the existence of (81), (88) and (92) for the 1-gap solution. Let

$$
\psi=e^{x \zeta(z)} \frac{\sigma(x-z+\kappa)}{\sigma(x+\kappa) \sigma(z-\kappa)} \quad \psi^{\dagger}=e^{-x \zeta(z)} \frac{\sigma(x+z+\kappa)}{\sigma(x+\kappa) \sigma(z+\kappa)}
$$

be solutions to

$$
\left(\partial^{2}+u\right) \psi=\left(\partial^{2}-2 \wp(x+\kappa)\right) \psi=\wp(z) \psi
$$


Then

$$
\begin{gathered}
\psi^{\dagger} \psi=\frac{\sigma(x-z+\kappa) \sigma(x+z+\kappa)}{\sigma^{2}(x+\kappa) \sigma(z-\kappa) \sigma(z+\kappa)}=\frac{\sigma^{2}(z)}{\sigma(z+\kappa) \sigma(z-\kappa)}(\wp(z)-\wp(x+\kappa)) \\
\left\langle\psi^{\dagger} \psi\right\rangle==\frac{\sigma^{2}(z)}{\sigma(z+\kappa) \sigma(z-\kappa)}(\wp(z)-\langle\wp(x+\kappa)\rangle)
\end{gathered}
$$

and let us take the average over a period $2 \tilde{\omega}$ to be $\langle\wp(x+\kappa)\rangle=2 \tilde{\eta}$. Also

$$
\begin{gathered}
d p=d(\zeta(z)+\log \sigma(2 \tilde{\omega}-z+\kappa)-\log \sigma(\kappa-z))=-d z(\wp(z)+\zeta(2 \tilde{\omega}-z+\kappa)-\zeta(\kappa-z))= \\
=-d z(\wp(z)-2 \tilde{\eta})
\end{gathered}
$$

and

$$
\begin{gathered}
\frac{d p}{\left\langle\psi^{\dagger} \psi\right\rangle}=d z \frac{\sigma(z+\kappa) \sigma(z-\kappa)}{\sigma^{2}(z)} \\
d \Omega=\frac{d p}{\left\langle\psi^{\dagger} \psi\right\rangle} \psi^{\dagger} \psi=d z \frac{\sigma(x+z+\kappa) \sigma(x+z-\kappa)}{\sigma^{2}(z) \sigma^{2}(x+\kappa)}=d z(\wp(z)-\wp(x+\kappa))
\end{gathered}
$$

Now, the variation $\tilde{\delta}$ explicitly looks as

$$
\begin{gathered}
\tilde{\delta} d \Omega \equiv \frac{d p}{\left\langle\psi^{\dagger} \psi\right\rangle} \psi_{\kappa}^{\dagger} \psi_{\kappa+\delta \kappa}=d z \frac{\sigma(z+\kappa) \sigma(z-\kappa) \sigma(x+z+\kappa) \sigma(x-z+\kappa+\delta \kappa)}{\sigma^{2}(z) \sigma(x+\kappa) \sigma(z+\kappa) \sigma(x+\kappa+\delta \kappa) \sigma(z-\kappa-\delta \kappa)}= \\
=d z \frac{\sigma(x+\kappa+z) \sigma(x+\kappa-z)}{\sigma^{2}(z) \sigma^{2}(x+\kappa)}\left[1+\delta \kappa(\zeta(x-z+\kappa)+\zeta(z-\kappa)-\zeta(x+\kappa))+\mathcal{O}\left((\delta \kappa)^{2}\right)\right] \\
=d z(\wp(z)-\wp(x+\kappa))\left[1+\delta \kappa(\zeta(x-z+\kappa)+\zeta(z-\kappa)-\zeta(x+\kappa))+\mathcal{O}\left((\delta \kappa)^{2}\right)\right]
\end{gathered}
$$

It is easy to see that (99) has non-zero residues at $z=0$ and $z=\kappa$ (the residue at $z=x+\kappa$ is suppressed by $\wp(z)-\wp(x+\kappa)$. They give

$$
\begin{aligned}
\operatorname{res}_{z=0} \delta d \Omega \sim & \delta \kappa \oint_{z \hookrightarrow 0} d z \wp(z)(\zeta(x-z+\kappa)+\zeta(z-\kappa)) \sim \delta \kappa \oint_{z \hookrightarrow 0} \zeta(z) d(\zeta(x-z+\kappa)+ \\
& +\zeta(z-\kappa)) \sim \delta \kappa(\wp(x+\kappa)+\wp(\kappa)) \sim \delta(\zeta(x+\kappa)+\zeta(\kappa)) \equiv \delta v(x)
\end{aligned}
$$

and

$$
\operatorname{res}_{z=\kappa} \delta d \Omega=\delta \kappa(\wp(\kappa)-\wp(x+\kappa))=d \Omega(\kappa)
$$

which follows from the comparison to 98 ).

The quantization of the symplectic structure $(75)$ is known to correspond to the complete description of the effective theory (not only its low-energy part) at least in the simplest case when $E=W(\mu)$ and $p=Q(\mu)$ were two functions (polynomials) on a complex sphere. The corresponding generating function (4) was essential in the definition of the duality transformation between two dual points with completely different behaviour (see 40 for details). The exact answer for the partition function $\log \mathcal{T}=\log \mathcal{T}_{0}+\log \mathcal{T}_{\theta} \equiv \mathcal{F}+\log \mathcal{T}_{\theta} \operatorname{should}$ also include the deformation of the oscillating part, corresponding to the massive excitations.

\section{Whitham equations}

In this section I am going to point out that there exists also another definition of the generating 1-form (4). This definition goes back to the general approach to construction of the effective actions which is known as the Bogolyubov-Whitham averaging method (see [29, 43, 44, for a comprehensive review and references). Though the Whitham dynamics is describes the commutative flows on the moduli spaces, averaging over the Jacobian - 
the fast part of the theory, its explicit formulation is most simple and natural in terms of connections on spectral curves [29, 43]. This definition has an advantage that it identifies (5) with the logariphm of the $\tau$-function of the Whitham hierarchy.

\subsection{Whitham equations}

Let us first remind the most general definition of the Whitham hierarchy given by Krichever [29, 43]. One has a local system of functions $\Omega_{A}$ on one-dimensional complex curve and the corresponding set of parameters $t_{A}$ so that it is possible to introduce a 1 -form in the space with co-ordinates $\{\lambda, t\}$ where $\lambda$ is a local parameter on a curve 11

$$
\omega=\sum \Omega_{A} \delta t_{A}
$$

The Whitham equations are

$$
\delta \omega \wedge \delta \omega=0 \quad \delta \omega=\partial_{\lambda} \Omega_{A} \delta \lambda \wedge \delta t_{A}+\partial_{B} \Omega_{A} \delta t_{A} \wedge \delta t_{B}
$$

so that one needs to check the independent vanishing of the two different terms $-\delta t^{4}$ and $\delta t^{3} \delta \lambda$. The second term gives

$$
\sum \partial_{\lambda} \Omega_{A} \partial_{B} \Omega_{C}=0
$$

for the antisymmetrized sum or introducing explicit co-ordinates

$$
t_{A_{0}} \equiv x \quad \Omega_{A_{0}}(\lambda, t) \equiv p(\lambda, t)
$$

adjusted to the fixed choice of parameters $\left\{t_{A}\right\}$

$$
\partial_{A} \Omega_{B}-\partial_{B} \Omega_{A}+\left\{\Omega_{A}, \Omega_{B}\right\}=0 \quad\left\{\Omega_{A}, \Omega_{B}\right\} \equiv \frac{\partial \Omega_{A}}{\partial x} \frac{\partial \Omega_{B}}{\partial p}-\frac{\partial \Omega_{B}}{\partial x} \frac{\partial \Omega_{A}}{\partial p}
$$

In fact (106) strongly depend on the choice of the local co-ordinate $p$. The equations (103), (104) and (106) are defined only locally and have huge amount of solutions.

A possible way to restrict ourselves is to get "globalized picture" related with the "modulation" of parameters of the finite-gap solutions of integrable systems of KP/Toda type. The KP $\tau$-function associated with a given spectral curve is

$$
\mathcal{T}\left\{t_{i}\right\}=e^{\sum t_{i} \gamma_{i j} t_{j}} \vartheta\left(\mathbf{\Phi}_{0}+\sum t_{i} \mathbf{k}_{i}\right) \quad \mathbf{k}_{i}=\oint_{\mathbf{B}} d \Omega_{i}
$$

where $\vartheta$ is a Riemann theta-function and $d \Omega_{i}$ are meromorphic 1-differentials with poles of the order $i+1$ at a marked point $z_{0}$. They are completely specified by the normalization relations

$$
\oint_{\mathbf{A}} d \Omega_{i}=0
$$

and the asymptotic behaviour

$$
d \Omega_{i}=\left(\xi^{-i-1}+o(\xi)\right) d \xi
$$

\footnotetext{
${ }^{11}$ Actually it might be better to write

$$
\tilde{\omega}=\omega+\Omega_{\lambda} d \lambda
$$
}

and consider $\Omega_{\lambda}=1$ as a sort of "gauge condition". 
where $\xi$ is a local coordinate in the vicinity of $z_{0}$. The moduli $\left\{u_{\alpha}\right\}$ of spectral curve are invariants of KP flows,

$$
\frac{\partial u_{\alpha}}{\partial t_{i}}=0
$$

The way the moduli depend on $t_{i}$ after the "modulation" is defined by the Whitham equations (106). For $z=z(\lambda, t)$ so that $\partial_{i} z=\left\{\Omega_{i}, z\right\}$ they acquire more simple form

$$
\frac{\partial d \Omega_{i}(z)}{\partial t_{j}}=\frac{\partial d \Omega_{j}(z)}{\partial t_{i}} .
$$

and imply that

$$
d \Omega_{i}(z)=\frac{\partial d S(z)}{\partial t_{i}}
$$

and the equations for moduli, following from (111), are:

$$
\frac{\partial u_{\alpha}}{\partial t_{i}}=v_{i j}^{\alpha \beta}(u) \frac{\partial u_{\beta}}{\partial t_{j}}
$$

with some (in general complicated) functions $v_{i j}^{\alpha \beta}$.

In the KdV (and Toda-chain) case all the spectral curves are hyperelliptic, and for the KdV $i$ takes only odd values $i=2 j+1$, so that

$$
d \Omega_{2 j+1}(z)=\frac{\mathcal{P}_{j+g}(z)}{y(z)} d z
$$

the coefficients of the polynomials $\mathcal{P}_{j}$ being fixed by normalization conditions (108), (109) (one usually takes $z_{0}=\infty$ and the local parameter in the vicinity of this point is $\xi=z^{-1 / 2}$ ). In this case the equations (113) can be diagonalized if the co-ordinates $\left\{u_{\alpha}\right\}$ on the moduli space are taken to be the ramification points:

$$
v_{i j}^{\alpha \beta}(u)=\left.\delta^{\alpha \beta} \frac{d \Omega_{i}(z)}{d \Omega_{j}(z)}\right|_{z=u_{\alpha}}
$$

Finally, the differential $d S(z)(112$ can be constructed for any finite-gap solution 24] and it coincides with the generating 1-form (4). The equality

$$
\frac{\partial \mathcal{F}}{\partial \mathbf{a}}=\mathbf{a}_{D} \quad \mathbf{a}=\oint_{\mathbf{A}} d S \quad \mathbf{a}_{D}=\oint_{\mathbf{B}} d S
$$

defines $\tau$-function of the Whitham hierarchy $\mathcal{F}=\log \mathcal{T}_{\text {Whitham }}$ 43. Below, the explicit examples of the Whitham solutions will be considered.

\subsection{Spherical solutions and topological gravity}

Let us, first, turn to the simplest nontrivial solutions of the systems (106), (111) related with $2 d$ topological string theories given at least locally by the formulas

$$
z(\lambda, t)^{p}=\lambda^{p}+u_{p-2}(t) \lambda^{p-2}+\ldots+u_{0}(t)
$$

so that

$$
\Omega_{i}(\lambda, t)=z(\lambda, t)_{+}^{i} \quad \Omega_{i}(z, t)=z^{i}+O\left(z^{\frac{i}{n}-1}\right)
$$

and in the $p=2$ example

$$
\begin{gathered}
z^{2}=\lambda^{2}+U(x, t) \quad \Omega_{i}(\lambda, t)=z(\lambda, t)_{+}^{i}=\left(\lambda^{2}+U(x, t)\right)_{+}^{\frac{1}{2}} \\
\Omega_{i}(z, t)=z^{i}+O\left(z^{\frac{i}{2}-1}\right)
\end{gathered}
$$


Now, in the simplest "global picture" it is possible to interpret $\lambda$ and $z$ as global co-ordinates on sphere with one marked point (where $\lambda=z=\infty$ ).

For particular choice of parameters $\left\{t_{k}\right\}$ this generic picture gives Whitham solutions coming from the $\mathrm{KP} /$ Toda equations in the following way. Starting with the "zero-gap" solution to the KdV hierarchy

$$
\begin{gathered}
U(x, t)=u=\text { const } \quad \Psi(\lambda, t)=e^{\sum_{k>0} t_{k} z^{k}(\lambda)_{+}} \\
\left(\partial_{t_{1}}^{2}+u\right) \Psi=z^{2} \Psi \quad \partial_{t_{k}} \Psi=\left(\partial_{t_{1}}^{2}+u\right)_{+}^{\frac{k}{2}} \Psi
\end{gathered}
$$

one comes by

$$
\begin{gathered}
\Omega_{1}=\overline{\log \Psi(\lambda, t)_{t_{1}}}=z(\lambda)_{+}=\lambda=\sqrt{z^{2}-u} \equiv p(z) \\
\Omega_{i}=\overline{\log \Psi(\lambda, t)_{t_{i}}}=z^{i}(\lambda)_{+}=\lambda^{3}+\frac{3}{2} u \lambda=\left(z^{2}-u\right)^{\frac{3}{2}}+\frac{3}{2} u \sqrt{z^{2}-u}
\end{gathered}
$$

to the formulas 119). The generating differential (112) is now

$$
d S(z)=\sum t_{k} d \Omega_{k}(z)
$$

and the solution can be found in terms of the "periods"

$$
t_{k}=\frac{1}{k} \operatorname{res}_{\infty}\left(z^{-k} d S\right)
$$

For example the third KdV flow gives rise to

$$
\partial_{t_{3}} \Omega_{1}(z)=\partial_{t_{1}} \Omega_{3}(z)
$$

which is equivalent to the Hopf or dispersionless KdV equation

$$
u_{t_{3}}-\frac{3}{2} u u_{t_{1}}=0
$$

with generic solution

$$
t_{1}+\frac{3}{2} t_{3} u+P(u)=0 \quad P(u) \sim \sum t_{2 n+1} u^{n}
$$

Now, for the purposes of quantum field theory one needs to take from (126) the solution of the Whitham equation which is exact solution of the "full" KdV

$$
u=-\frac{2}{3} \frac{t_{1}}{t_{3}}
$$

The generating differential (112) is now

$$
\left.d S\right|_{t_{3}=\frac{2}{3}, t_{k \neq 3}=0}=\lambda d\left(z^{2}(\lambda)\right)=-\left(\lambda^{2}+u\right) d \lambda+d(\ldots)
$$

Then the solution to the linear problem

$$
\left(\partial_{t_{1}}^{2}-\frac{2}{3} \frac{t_{1}}{t_{3}}\right) \Psi=z^{2} \Psi
$$

gives

$$
\begin{gathered}
\left.\Psi\left(t_{1}, z\right)\right|_{t_{3}=\frac{2}{3}}=A i\left(t_{1}+z^{2}\right) \\
\phi_{i}(z) \sim \frac{\partial^{i-1} \Psi(z, t)}{\partial t_{1}^{i-1}}=\sqrt{2 z} e^{-\frac{2}{3} z^{3}} \int d x x^{i-1} e^{-\frac{x^{3}}{3}+x z^{2}}
\end{gathered}
$$

and the determinant formula

$$
\frac{\mathcal{T}(t+T)}{\mathcal{T}(t)}=\frac{\operatorname{det} \phi_{i}\left(z_{j}\right)}{\Delta(z)}
$$

results in the $\tau$-function of the whole hierarchy in Miwa co-ordinates $T_{k}=-\frac{1}{k} \sum z_{j}^{-k}$. The decomposition of $\log \mathcal{T}(T)$ gives the correlators (1) of two-dimensional topological gravity. 


\subsection{Elliptic curves and the Gurevich-Pitaevsky solutions}

Now let us demonstrate that the higher genus Riemann surfaces (already in the elliptic case) give rise to nonperturbative formulation of physically less trivial theories. In contrast to the previous example Whitham times will be nontrivially related to the moduli of the curve. For example, from a standard definition (see [29, 43]) in case of the elliptic curve $y^{2}=\prod_{i=1}^{3}\left(x-u_{i}\right)$ one has

$$
t_{k}=\frac{2}{k(2-k)} \operatorname{res}\left(x^{1-\frac{k}{2}} d y\right)
$$

or substituting $x=\wp(\xi)+c$ and $y=\frac{1}{2} \wp^{\prime}(\xi)$, where $\wp(\xi)$ is the Weierstrass $\wp$-function,

$$
\begin{gathered}
t_{k}=-\frac{2}{k(2-k)} \operatorname{res}\left((\wp(\xi)+c)^{1-\frac{k}{2}} \wp^{\prime \prime}(\xi) d \xi\right)= \\
=-\frac{6}{k(2-k)} \operatorname{Res} \frac{d \xi}{\xi^{6-k}}\left(1+c \xi^{2}+\frac{g_{2}}{20} \xi^{4}+\ldots\right)^{1-\frac{k}{2}}\left(1+\frac{g_{2}}{60} \xi^{4}+\ldots\right)= \\
=\frac{2}{5} \delta_{k, 5}-c \delta_{k, 3}+\left(\frac{3}{4} c^{2}-\frac{1}{4} g_{2}\right) \delta_{k, 1}+\mathcal{O}\left(t_{-k}\right)
\end{gathered}
$$

with dependent negative times $t_{-k}=t_{-k}\left(t_{1}, t_{3}\right)$ [0].

The elliptic solution to the KdV is

$$
\begin{aligned}
& U\left(t_{1}, t_{3}, \ldots \mid u\right)=\frac{\partial^{2}}{\partial t_{1}^{2}} \log \mathcal{T}\left(t_{1}, t_{3}, \ldots \mid u\right)= \\
& =U_{0 \wp}\left(k_{1} t_{1}+k_{3} t_{3}+\ldots+\Phi_{0} \mid \omega, \omega^{\prime}\right)+\frac{u}{3}
\end{aligned}
$$

and

$$
\begin{gathered}
d p \equiv d \Omega_{1}(z)=\frac{z-\alpha(u)}{y(z)} d z, \\
d Q \equiv d \Omega_{3}(z)=\frac{z^{2}-\frac{1}{2} u z-\beta(u)}{y(z)} d z .
\end{gathered}
$$

Normalization conditions (108) prescribe that

$$
\alpha(u)=\frac{\oint_{A} \frac{z d z}{y(z)}}{\oint_{A} \frac{d z}{y(z)}} \quad \beta(u)=\frac{\oint_{A} \frac{\left(z^{2}-\frac{1}{2} u z\right) d z}{y(z)}}{\oint_{A} \frac{d z}{y(z)}} .
$$

The simplest elliptic example is the first Gurevich-Pitaevsky (GP) solution 45 with the underlying spectral curve

$$
y^{2}=\left(z^{2}-1\right)(z-u)
$$

specified by a requirement that all branching points except for $z=u$ are fixed and do not obey Whitham deformation. It is easy to see that by change of variables $z=u+\lambda^{2}$ and $y \rightarrow y \lambda$ the curve (137) can be written as (a particular $N_{c}=2$ case) of the Toda-chain curve (18) $y^{2}=\left(\lambda^{2}+u\right)^{2}-1$ c $P_{N_{c}=2}(\lambda)=\lambda^{2}+h_{2}$ i.e. $h_{2} \equiv u$. The generating differential (何), 112, corresponding to (137) is given by [9]

$$
d S(z)=\left(t_{1}+t_{3}\left(z+\frac{1}{2} u\right)+\ldots\right) \frac{z-u}{y(z)} d z \underset{\left\{t_{k>1}=0\right\}}{=} t_{1} \frac{z-u}{y(z)} d z
$$

and it produces the simplest solution to (111) coming from the elliptic curve. From (138) one derives:

$$
\begin{gathered}
\frac{\partial d S(z)}{\partial t_{1}}=\left(z-u-\left(\frac{1}{2} t_{1}+u t_{3}\right) \frac{\partial u}{\partial t_{1}}\right) \frac{d z}{y(z)}, \\
\frac{\partial d S(z)}{\partial t_{3}}=\left(z^{2}-\frac{1}{2} u z-\frac{1}{2} u^{2}-\left(\frac{1}{2} t_{1}+u t_{3}\right) \frac{\partial u}{\partial t_{3}}\right) \frac{d z}{y(z)},
\end{gathered}
$$


and comparison with explicit expressions 135 implies:

$$
\begin{gathered}
\left(\frac{1}{2} t_{1}+u t_{3}\right) \frac{\partial u}{\partial t_{1}}=\alpha(u)-u, \\
\left(\frac{1}{2} t_{1}+u t_{3}\right) \frac{\partial u}{\partial t_{3}}=\beta(u)-\frac{1}{2} u^{2} .
\end{gathered}
$$

In other words, this construction provides the first GP solution to the Whitham equation

$$
\frac{\partial u}{\partial t_{3}}=v_{31}(u) \frac{\partial u}{\partial t_{1}}
$$

with

$$
v_{31}(u)=\frac{\beta(u)-\frac{1}{2} u^{2}}{\alpha(u)-u}=\left.\frac{d \Omega_{3}(z)}{d \Omega_{1}(z)}\right|_{z=u},
$$

which can be expressed through elliptic integrals 45$]$.

The elliptic solution with all moving branch points is given instead of (138) by

$$
y^{2}=\prod_{i=1}^{3}\left(z-u_{i}\right) \quad d S=y(z) d z
$$

and all $u_{i}$ are some functions of the Whitham times. Then one has to fulfil

$$
\begin{gathered}
d p=\frac{\partial}{\partial t_{1}} d S=y d z \sum_{i=1}^{3} \frac{v_{i}}{z-u_{i}}=\frac{z-\alpha(u)}{y} d z \\
d Q=\frac{\partial}{\partial t_{3}} d S=y d z \sum_{i=1}^{3} \frac{w_{i}}{z-u_{i}}=\frac{z^{2}-\left(\frac{1}{2} \sum u_{i}\right) z-\beta(u)}{y} d z
\end{gathered}
$$

where

$$
v_{i}=-\frac{1}{2} \frac{\partial u_{i}}{\partial t_{1}} \quad w_{i}=-\frac{1}{2} \frac{\partial u_{i}}{\partial t_{3}}
$$

and $\alpha(u)$ and $\beta(u)$ are defined as before. Now what one gets is a simple linear system of the equations

$$
\begin{gathered}
R_{i j}(u) v_{j}=V_{i} \\
R_{i j}(u)=\left(\begin{array}{ccc}
1 & 1 & 1 \\
u_{2}+u_{3} & u_{1}+u_{3} & u_{1}+w_{2} \\
u_{2} u_{3} & u_{1} u_{3} & u_{1} u_{2}
\end{array}\right) \\
V^{T}=(0,-1,-\alpha(u)) \quad W^{T}=\left(1, \frac{1}{2}\left(u_{1}+u_{2}+u_{3}\right),-\beta(u)\right)
\end{gathered}
$$

The solution of linear system looks similar to (141), for example:

$$
\frac{w_{1}}{v_{1}}=\frac{u_{1}^{2}-\frac{1}{2}\left(u_{1}+u_{2}+u_{3}\right)-\beta(u)}{u_{1}-\alpha(u)}
$$

and this class of solutions contain, for example, pure (non-topological!) $2 d$ gravity. The second GP solution 12

$$
y^{2}=\prod_{i=1}^{3}\left(z-u_{i}\right) \quad d S=(z-e) y d z
$$

can be obtained from elliptic curve with a marked point. In all these cases, one might expect that a "modulated" $\tau$-function (cf. with (107)) would still have a form

$$
\mathcal{T}\left\{t_{i}\right\}=e^{\mathcal{F}(t)} \vartheta(\mathbf{S}(t)) \equiv \mathcal{T}_{\text {Whitham }} \mathcal{T}_{\Theta}
$$

\footnotetext{
${ }^{12}$ corresponding to the Yang-Lee edge singularity or $(2,5)$ conformal minimal model interacting with $2 d$ gravity
} 
so that

$$
\mathbf{k}_{i}(t)=\frac{\partial \mathbf{S}}{\partial t_{i}}
$$

and the poles of the "effective" potential

$$
U(t)=\frac{\partial^{2}}{\partial t_{1}^{2}} \log \mathcal{T}
$$

can be identified with the massive excitations (8).

For the higher genus curves the above procedure looks to be exactly the same [9, 46, 47]. The only problem is in explicit formulas which look nontrivially already in the GP case, while the formal definitions (四), (112) are again formulated in terms of the eigenvalues of two operators.

\section{$5 \quad$ Prepotential of the Effective Theory}

In the previous section the prepotential $\mathcal{F}$ was identified with the logariphm of the $\tau$-function of the Whitham hierarchy. Such identification, being a particular case of the fundamental formula relating the generating functions or effective actions of quantum theories to the $\tau$-functions of the hierarchies of integrable equations, has a little bit implicit form. In this section, I am will formulate and discuss explicitely a system of differential equations satisfied by the prepotential $\mathcal{F}$. Following [50] the proof of these equations for the $\mathcal{N}=2$ SUSY gluodynamics will be presented, the case of $\mathcal{N}=2$ Yang-Mills theory with matter will be discussed in detail in a forthcoming paper 51 .

\subsection{The associativity equations}

The prepotential $\mathcal{F}$ [6, 7] is defined in terms of a family of Riemann surfaces, endowed with the meromorphic differential $d S$. For the gauge group $G=S U(N)$ the family is [6, 7], [21, 9] given by (17) and the generating differential by (㺼). The prepotential $\mathcal{F}\left(a_{i}\right)$ is implicitly defined by the set of equations (116). According to [9], this definition identifies $\mathcal{F}\left(a_{i}\right)$ as logarithm of (truncated) $\tau$-function of Whitham integrable hierarchy. Existing experience with Whitham hierarchies [105] 29, 30] implies that $\mathcal{F}\left(a_{i}\right)$ should satisfy some sort of the Witten-Dijkgraaf-Verlinde-Verlinde (WDVV) equations [27]. Below in this section we demonstrate that WDVV equations for the prepotential actually look like

$$
\mathcal{F}_{i} \mathcal{F}_{k}^{-1} \mathcal{F}_{j}=\mathcal{F}_{j} \mathcal{F}_{k}^{-1} \mathcal{F}_{i} \quad \forall i, j, k=1, \ldots, N-1
$$

Here $\mathcal{F}_{i}$ denotes the matrix

$$
\left(\mathcal{F}_{i}\right)_{m n}=\frac{\partial^{3} \mathcal{F}}{\partial a_{i} \partial a_{m} \partial a_{n}}
$$

Few comments are now in order:

(i). Let us remind, first, that the conventional WDVV equations for topological field theory express the associativity of the algebra $\phi_{i} \phi_{j}=C_{i j}^{k} \phi_{k}$ (for symmetric in $i$ and $j$ structure constants): $\left(\phi_{i} \phi_{j}\right) \phi_{k}=\phi_{i}\left(\phi_{j} \phi_{k}\right)$, or $C_{i} C_{j}=C_{j} C_{i}$, for the matrix $\left(C_{i}\right)_{n}^{m} \equiv C_{i n}^{m}$. These conditions become highly non-trivial since, in topological theory, the structure constants are expressed in terms of a single prepotential $\mathcal{F}\left(t_{i}\right): C_{i j}^{l}=\left(\eta_{(0)}^{-1}\right)^{k l} \mathcal{F}_{i j k}$, and $\mathcal{F}_{i j k}=\frac{\partial^{3} \mathcal{F}}{\partial t_{i} \partial t_{j} \partial t_{k}}$, while the metric is $\eta_{k l}^{(0)}=\mathcal{F}_{0 k l}$, where $\phi_{0}=I$ is the unity operator. In other words, the 
conventional WDVV equations can be written as

$$
\mathcal{F}_{i} \mathcal{F}_{0}^{-1} \mathcal{F}_{j}=\mathcal{F}_{j} \mathcal{F}_{0}^{-1} \mathcal{F}_{i}
$$

In contrast to (152), $k$ is restricted to $k=0$, associated with the distinguished unity operator.

On the other hand, in the Seiberg-Witten theory there does not clearly exist any distinguished index $i$ : all the arguments $a_{i}$ of the prepotential are on equal footing. Thus, if some kind of the WDVV equations holds in this case, it should be invariant under any permutation of indices $i, j, k$-criterium satisfied by the system (152). Moreover, the same set of equations (152) is satisfied for generic topological theory.

(ii). In the general theory of Whitham hierarchies [29, 30] the WDVV equations arise also in the form (154). Again, there exists a distinguished time-variable (105) for the global solutions usually associated with the first time-variable of the original $\mathrm{KP} / \mathrm{KdV}$ hierarchy. Moreover, usually - in contrast to the simplest topological models - the set of these variables for the Whitham hierarchy is infinitely large. In this context our eqs.(152) state that, for specific subhierarchies (in the Seiberg-Witten gluodynamics, it is the Toda-chain hierarchy, associated with a peculiar set of hyperelliptic surfaces), there exists a non-trivial truncation of the quasiclassical $\tau$-function, when it depends on the finite number $(N-1=g$ - genus of the Riemann surface) of equivalent arguments $a_{i}$, and satisfies a much wider set of WDVV-like equations: the whole set (152).

(iii). From (116) it is clear that $a_{i}$ 's are defined modulo linear transformations (one can change $A$-cycle for any linear combination of them). Eqs.(152) possess adequate "covariance": the least trivial part is that $\mathcal{F}_{k}$ can be substituted by $\mathcal{F}_{k}+\sum_{l} \epsilon_{l} \mathcal{F}_{l}$. Then

$$
\mathcal{F}_{k}^{-1} \rightarrow\left(\mathcal{F}_{k}+\sum \epsilon_{l} \mathcal{F}_{l}\right)^{-1}=\mathcal{F}_{k}^{-1}-\sum \epsilon_{l} \mathcal{F}_{k}^{-1} \mathcal{F}_{l} \mathcal{F}_{k}^{-1}+\sum \epsilon_{l} \epsilon_{l} \mathcal{F}_{k}^{-1} \mathcal{F}_{l} \mathcal{F}_{k}^{-1} \mathcal{F}_{l^{\prime}} \mathcal{F}_{k}^{-1}+\ldots
$$

Clearly, (152) - valid for all triples of indices simultaneously - is enough to guarantee that $\mathcal{F}_{i}\left(\mathcal{F}_{k}+\sum \epsilon_{l} \mathcal{F}_{l}\right)^{-1} \mathcal{F}_{j}=$ $\mathcal{F}_{j}\left(\mathcal{F}_{k}+\sum \epsilon_{l} \mathcal{F}_{l}\right)^{-1} \mathcal{F}_{i}$. Covariance under any replacement of $A$ and $B$-cycles together will be seen from the general proof below: in fact the role of $\mathcal{F}_{k}$ can be played by $\mathcal{F}_{d \omega}$, associated with any holomorphic 1-differential $d \omega$ on the Riemann surface.

(iv). For metric $\eta$, which is a second derivative,

$$
\eta_{i j}=\frac{\partial^{2} h}{\partial a_{i} \partial a_{j}} \equiv h_{, i j}
$$

(as is the case for our $\left.\left.\eta_{m n}^{(k)} \equiv\left(\mathcal{F}_{k}\right)_{m n}\right): \quad h=h^{(k)}=\partial \mathcal{F} / \partial a_{k}\right), \Gamma_{j k}^{i}=\frac{1}{2} \eta^{i m} h_{, j k m}$ and the Riemann tensor

$$
\begin{aligned}
R_{j k l}^{i}=\Gamma_{j l, k}^{i}+ & \Gamma_{k n}^{i} \Gamma_{j l}^{n}-(k \leftrightarrow l)=\frac{1}{2} \eta^{i m} h_{, j k l m}-\frac{1}{4} \eta^{i p} h_{, p n k} \eta^{n m} h_{, m j l}+-(k \leftrightarrow l)= \\
& =-\Gamma_{k n}^{i} \Gamma_{j l}^{n}+(k \leftrightarrow l)=-\frac{1}{4} \eta^{i m} h_{, m n j} \eta^{n p} h_{, p k l}+(k \leftrightarrow l)
\end{aligned}
$$

In terms of the matrix $\eta=\left\{(\eta)_{k l}\right\}$ the zero-curvature condition $R_{i j k l}=0$ would be

$$
\eta_{, i} \eta^{-1} \eta_{, j} \stackrel{?}{=} \eta, j \eta^{-1} \eta_{, i}
$$

This equation is remarkably similar to (152) and (154), but when $\eta_{i j}^{(k)}=\mathcal{F}_{i j k}$ is substituted into (158), it contains the fourth derivatives of $\mathcal{F}$ :

$$
\mathcal{F}_{k, i} \mathcal{F}_{k}^{-1} \mathcal{F}_{k, j}=\mathcal{F}_{k, j} \mathcal{F}_{k}^{-1} \mathcal{F}_{i, k} \quad \forall i, j, k=1, \ldots, N-1
$$


(no summation over $k$ in this formula!), while (152) is expressed through the third derivatives only.

In ordinary topological theories $\eta^{(0)}$ is always flat, i.e. (159) holds for $k=0$ along with (154) - and this allows one to choose "flat coordinates" where $\eta^{(0)}=$ const. Sometimes - see the example below - all the metrics $\eta^{(k)}$ are flat simultaneously. However, this is not always the case: in the example of quantum cohomologies of $C P^{2}$ [4, 48] eqs.(152) are true for all $k=0,1,2$, but only $\eta^{(0)}$ is flat (satisfies (158)), while $\eta^{(1)}$ and $\eta^{(2)}$ lead to non-vanishing curvatures.

(v). It is well known that the conventional WDVV equations (154) are pretty restrictive (at least in the case of so called semisimple Frobenius manifolds in the terminology of [30, 48] which however do not include one of the most interesting examples of quantum cohomologies of the Calabi-Yau type manifolds with the vanishing first Chern class): this is an overdetermined system of equations for a single function $\mathcal{F}\left(t_{i}\right)$, and it is a kind of surprise that they possess any solutions at all, and in fact there exist vast variety of them (associated with Whitham hierarchies, topological models and quantum cohomologies). The set (152) is even more overdetermined than (154), since $k$ can take any value. Thus, it is even more surprising that the solutions still exist (in order to convince the reader, we supplement the formal proof by explicit examples). Of course, (152) is tautologically true for $N=2$ and $N=3$, it becomes a non-trivial system for $N \geq 4$.

(vi). Our consideration suggests that when the ordinary WDVV (154) is true, the whole system (152) holds automatically for any other $k$ (with the only restriction that $\mathcal{F}_{k}$ is non-degenerate). Indeed, ${ }^{13}$

$$
\mathcal{F}_{i} \mathcal{F}_{k}^{-1} \mathcal{F}_{j}=\mathcal{F}_{0}\left(C_{i}^{(0)}\left(C_{k}^{(0)}\right)^{-1} C_{j}^{(0)}\right)
$$

is obviously symmetric w.r.to the permutation $i \leftrightarrow j$ implied by $\left[C_{i}^{(0)}, C_{j}^{(0)}\right]=0$.

This implies that this entire system should possess some interpretation in the spirit of hierarchies or hidden symmetries. It still remains to be found. The geometrical or cohomological origin of relations (152) also remains obscure.

(vii). One can also look for relation between (152) and Picard-Fuchs equations, and then address to the issue of the WDVV equations for the prepotential, associated with families of the Calabi-Yau manifolds.

(viii). Effective theory (116) is naively non-topological. From the 4-dimensional point of view it describes the low-energy limit of the Yang-Mills theory which - at least, in the $\mathcal{N}=2$ supersymmetric case - is not topological and contains propagating massless particles. Still this theory is entirely defined by a prepotential, which - as we now see - possesses all essential properties of the prepotentials in topological theory. Thus, from the "stringy" point of view (when everything is described in terms of universality classes of effective actions) the Seiberg-Witten models belong to the same class as topological models: only the way to extract physically meaningful correlators from the prepotential is different. This can serve as a new evidence that the notion of topological theories is deeper than it is usually assumed: as emphasized in [9] it can be actually more related to the low-energy (IR) limit of field theories than to the property of the correlation functions to be constants in physical space-time.

\footnotetext{
${ }^{13}$ This simple proof was suggested by A.Rosly.
} 


\subsection{The proof of the associativity equations}

Let us start with reminding the proof of the WDVV equations (154) for ordinary topological theories. We take the simplest of all possible examples, when $\phi_{i}$ are polynomials of a single variable $\lambda$. The proof is essentially the check of consistency between the following formulas:

$$
\begin{gathered}
\phi_{i}(\lambda) \phi_{j}(\lambda)=C_{i j}^{k} \phi_{k}(\lambda) \bmod W^{\prime}(\lambda), \\
\mathcal{F}_{i j k}=\operatorname{res} \frac{\phi_{i} \phi_{j} \phi_{k}(\lambda)}{W^{\prime}(\lambda)}=\sum_{\alpha} \frac{\phi_{i} \phi_{j} \phi_{k}\left(\lambda_{\alpha}\right)}{W^{\prime \prime}\left(\lambda_{\alpha}\right)}, \\
\eta_{k l}=\operatorname{res} \frac{\phi_{k} \phi_{l}(\lambda)}{W^{\prime}(\lambda)}=\sum_{\alpha} \frac{\phi_{k} \phi_{l}\left(\lambda_{\alpha}\right)}{W^{\prime \prime}\left(\lambda_{\alpha}\right)} \\
\mathcal{F}_{i j k}=\eta_{k l} C_{i j}^{l} .
\end{gathered}
$$

Here $\lambda_{\alpha}$ are the roots of $W^{\prime}(\lambda)$.

In addition to the consistency of (161)-(164), one should know that such $\mathcal{F}_{i j k}$, given by (162), are the third derivatives of a single function $\mathcal{F}(a)$, i.e.

$$
\mathcal{F}_{i j k}=\frac{\partial^{3} \mathcal{F}}{\partial a_{i} \partial a_{j} \partial a_{k}} .
$$

This integrability property of (162) follows from separate arguments and can be checked independently. But if (161)-(163) is given, the proof of (164) is straightforward:

$$
\begin{gathered}
\eta_{k l} C_{i j}^{l}=\sum_{\alpha} \frac{\phi_{k} \phi_{l}\left(\lambda_{\alpha}\right)}{W^{\prime \prime}\left(\lambda_{\alpha}\right)} C_{i j}^{l} \stackrel{(161)}{=} \\
=\sum_{\alpha} \frac{\phi_{k}\left(\lambda_{\alpha}\right)}{W^{\prime \prime}\left(\lambda_{\alpha}\right)} \phi_{i}\left(\lambda_{\alpha}\right) \phi_{j}\left(\lambda_{\alpha}\right)=\mathcal{F}_{i j k} .
\end{gathered}
$$

Note that (161) is defined modulo $W^{\prime}(\lambda)$, but $W^{\prime}\left(\lambda_{\alpha}\right)=0$ at all the points $\lambda_{\alpha}$. Imagine now that we change the definition of the metric:

$$
\eta_{k l} \rightarrow \eta_{k l}(\omega)=\sum_{\alpha} \frac{\phi_{k} \phi_{l}\left(\lambda_{\alpha}\right)}{W^{\prime \prime}\left(\lambda_{\alpha}\right)} \omega\left(\lambda_{\alpha}\right)
$$

Then the WDVV equations would still be correct, provided the definition (161) of the algebra is also changed for

$$
\phi_{i}(\lambda) \phi_{j}(\lambda)=C_{i j}^{k}(\omega) \phi_{k}(\lambda) \omega(\lambda) \bmod W^{\prime}(\lambda)
$$

This describes an associative algebra, whenever the polynomials $\omega(\lambda)$ and $W^{\prime}(\lambda)$ are co-prime, i.e. do not have common divisors. Note that $(162)$ - and thus the fact that $\mathcal{F}_{i j k}$ is the third derivative of the same $\mathcal{F}$ - remains intact! One can now take for $\omega(\lambda)$ any of the operators $\phi_{k}(\lambda)$, thus reproducing eqs. (152) for all topological theories 14 .

In the case of the Seiberg-Witten model the polynomials $\phi_{i}(\lambda)$ are substituted by the canonical holomorphic differentials $d \omega_{i}(\lambda)$ on hyperelliptic surface (17). This surface can be represented in a standard hyperelliptic form (18). Instead of (161) and (168) we now put

$$
d \omega_{i}(\lambda) d \omega_{j}(\lambda)=C_{i j}^{k}(d \omega) d \omega_{k}(\lambda) d \omega(\lambda) \bmod \frac{d P_{N}(\lambda) d \lambda}{y^{2}} .
$$

\footnotetext{
${ }^{14}$ To make $(152)$ sensible, one should require that $W^{\prime}(\lambda)$ has only simple zeroes, otherwise some of the matrices $\mathcal{F}_{k}$ can be degenerate and non-invertible.
} 
In contrast to (168) we can not now choose $\omega=1$ (to reproduce (161)), because now we need it to be a 1differential. Instead we just take $d \omega$ to be a holomorphic 1-differential. However, there is no distinguished one - just a $g$-parametric family - and $d \omega$ can be any one from this family. We require only that it is co-prime with $\frac{d P_{N}(\lambda)}{y}$.

If the algebra (169) exists, the structure constants $C_{i j}^{k}(d \omega)$ satisfy the associativity condition (if $d \omega$ and $\frac{d P_{N}}{y}$ are co-prime). But we still need to show that it indeed exists, i.e. that if $d \omega$ is given, one can find ( $\lambda$-independent) $C_{i j}^{k}$. This is a simple exercise: all $d \omega_{i}$ are linear combinations of

$$
\begin{gathered}
d v_{k}(\lambda)=\frac{\lambda^{k-1} d \lambda}{y}, \quad k=1, \ldots, g: \\
d v_{k}(\lambda)=\sigma_{k i} d \omega_{i}(\lambda), \quad d \omega_{i}=\left(\sigma^{-1}\right)_{i k} d v_{k}, \quad \sigma_{k i}=\oint_{A_{i}} d v_{k},
\end{gathered}
$$

also $d \omega(\lambda)=s_{k} d v_{k}(\lambda)$. Thus, 169 is in fact a relation between the polynomials:

$$
\left(\sigma_{i i^{\prime}}^{-1} \lambda^{i^{\prime}-1}\right)\left(\sigma_{j j^{\prime}}^{-1} \lambda^{j^{\prime}-1}\right)=C_{i j}^{k}\left(\sigma_{k k^{\prime}}^{-1} \lambda^{k^{\prime}-1}\right)\left(s_{l} \lambda^{l-1}\right)+p_{i j}(\lambda) P_{N}^{\prime}(\lambda) .
$$

At the l.h.s. we have a polynomial of degree $2(g-1)$. Since $P_{N}^{\prime}(\lambda)$ is a polynomial of degree $N-1=g$, this implies that $p_{i j}(\lambda)$ should be a polynomial of degree $2(g-1)-g=g-2$. The identification of two polynomials of degree $2(g-1)$ impose a set of $2 g-1$ equations for the coefficients. We have a freedom to adjust $C_{i j}^{k}$ and $p_{i j}(\lambda)$ (with $i, j$ fixed), i.e. $g+(g-1)=2 g-1$ free parameters: exactly what is necessary. The linear system of equations is non-degenerate for co-prime $d \omega$ and $d P_{N} / y$.

Thus, we proved that the algebra (169) exists (for a given $d \omega$ ) - and thus $C_{i j}^{k}(d \omega)$ satisfy the associativity condition

$$
C_{i}(d \omega) C_{j}(d \omega)=C_{j}(d \omega) C_{i}(d \omega) .
$$

Hence, instead of (162) we have [29, 30, 48]:

$$
\begin{gathered}
\mathcal{F}_{i j k}=\frac{\partial^{3} \mathcal{F}}{\partial a_{i} \partial a_{j} \partial a_{k}}=\frac{\partial T_{i j}}{\partial a_{k}}= \\
=\underset{d \lambda=0}{\operatorname{res}} \frac{d \omega_{i} d \omega_{j} d \omega_{k}}{d \lambda\left(\frac{d w}{w}\right)}=\underset{d \lambda=0}{\operatorname{res}} \frac{d \omega_{i} d \omega_{j} d \omega_{k}}{d \lambda \frac{d P_{N}}{y}}=\sum_{\alpha} \frac{\hat{\omega}_{i}\left(\lambda_{\alpha}\right) \hat{\omega}_{j}\left(\lambda_{\alpha}\right) \hat{\omega}_{k}\left(\lambda_{\alpha}\right)}{P_{N}^{\prime}\left(\lambda_{\alpha}\right) / \hat{y}\left(\lambda_{\alpha}\right)}
\end{gathered}
$$

The sum at the r.h.s. goes over all the $2 g+2$ ramification points $\lambda_{\alpha}$ of the hyperelliptic curve (i.e. over the zeroes of $\left.y^{2}=P_{N}^{2}(\lambda)-1=\prod_{\alpha=1}^{N}\left(\lambda-\lambda_{\alpha}\right)\right) ; \quad d \omega_{i}(\lambda)=\left(\hat{\omega}_{i}\left(\lambda_{\alpha}\right)+O\left(\lambda-\lambda_{\alpha}\right)\right) d \lambda, \quad \hat{y}^{2}\left(\lambda_{\alpha}\right)=\prod_{\beta \neq \alpha}\left(\lambda_{\alpha}-\lambda_{\beta}\right)$. Formula (173) can be extracted from [29], and its proof is presented in [50].

We define the metric in the following way:

$$
\begin{aligned}
\eta_{k l}(d \omega)= & \underset{d \lambda=0}{\operatorname{res}} \frac{d \omega_{k} d \omega_{l} d \omega}{d \lambda\left(\frac{d w}{w}\right)}=\underset{d \lambda=0}{\operatorname{res}} \frac{d \omega_{k} d \omega_{l} d \omega_{k}}{d \lambda \frac{d P_{N}}{y}}= \\
& =\sum_{\alpha} \frac{\hat{\omega}_{k}\left(\lambda_{\alpha}\right) \hat{\omega}_{l}\left(\lambda_{\alpha}\right) \hat{\omega}\left(\lambda_{\alpha}\right)}{P_{N}^{\prime}\left(\lambda_{\alpha}\right) / \hat{y}\left(\lambda_{\alpha}\right)}
\end{aligned}
$$

In particular, for $d \omega=d \omega_{k}, \eta_{i j}\left(d \omega_{k}\right)=\mathcal{F}_{i j k}$ : this choice will give rise to 152).

Given (169), 173) and (174), one can check:

$$
\mathcal{F}_{i j k}=\eta_{k l}(d \omega) C_{i j}^{k}(d \omega)
$$


Note that $\mathcal{F}_{i j k}=\frac{\partial^{3} \mathcal{F}}{\partial a_{i} \partial a_{j} \partial a_{k}}$ at the l.h.s. of $(175)$ is independent of $d \omega$ ! The r.h.s. of $(175)$ is equal to:

$$
\begin{gathered}
\eta_{k l}(d \omega) C_{i j}^{k}(d \omega)=\underset{d \lambda=0}{\operatorname{res}} \frac{d \omega_{k} d \omega_{l} d \omega}{d \lambda\left(\frac{d w}{w}\right)} C_{i j}^{l}(d \omega) \stackrel{(169)}{=} \\
=\operatorname{res} \frac{d \omega_{k}}{d \lambda\left(\frac{d w}{w}\right)}\left(d \omega_{i} d \omega_{j}-p_{i j} \frac{d P_{N} d \lambda}{y^{2}}\right)=\mathcal{F}_{i j k}-\underset{d \lambda=0}{\operatorname{res}} \frac{d \omega_{k}}{d \lambda\left(\frac{d P_{N}}{y}\right)} p_{i j}(\lambda) \frac{d P_{N} d \lambda}{y^{2}}= \\
=\mathcal{F}_{i j k}-\underset{d \lambda=0}{\operatorname{res}} \frac{p_{i j}(\lambda) d \omega_{k}(\lambda)}{y}
\end{gathered}
$$

It remains to prove that the last item is indeed vanishing for any $i, j, k$. This follows from the fact that $\frac{p_{i j}(\lambda) d \omega_{k}(\lambda)}{y}$ is singular only at zeroes of $y$, it is not singular at $\lambda=\infty$ because $p_{i j}(\lambda)$ is a polynomial of low enough degree $g-2<g+1$. Thus the sum of its residues at ramification points is thus the sum over all the residues and therefore vanishes.

This completes the proof of associativity condition for any $d \omega$. Taking $d \omega=d \omega_{k}$ (which is obviously co-prime with $\frac{d P_{N}}{y}$ ), we obtain (152).

\subsection{Explicit examples}

In this section it is demonstrated explicitly that the system (152) holds for the perturbative part of the SeibergWitten prepotential for $\mathcal{N}=2$ SUSY gluodynamics and for the quantum cohomologies of $C P^{2}$. Other examples can be found in [50].

Consider first the quantum cohomology of $C P^{2}$ 49. The prepotential is

$$
\mathcal{F}=\frac{1}{2} t_{0} t_{1}^{2}+\frac{1}{2} t_{0}^{2} t_{2}+\sum_{n=1}^{\infty} \frac{N_{n} t_{2}^{3 n-1}}{(3 n-1) !} e^{n t_{1}}
$$

and the corresponding matrices are:

$$
\mathcal{F}_{0}=\left(\begin{array}{ccc}
0 & 0 & 1 \\
0 & 1 & 0 \\
1 & 0 & 0
\end{array}\right) \quad \mathcal{F}_{1}=\left(\begin{array}{ccc}
0 & 1 & 0 \\
1 & \mathcal{F}_{111} & \mathcal{F}_{112} \\
0 & \mathcal{F}_{112} & \mathcal{F}_{122}
\end{array}\right) \quad \mathcal{F}_{2}=\left(\begin{array}{ccc}
1 & 0 & 0 \\
0 & \mathcal{F}_{112} & \mathcal{F}_{122} \\
0 & \mathcal{F}_{122} & \mathcal{F}_{222}
\end{array}\right)
$$

where

$$
\begin{array}{ll}
\mathcal{F}_{111}=\sum_{n} \frac{n^{3} N_{n}}{(3 n-1) !} t_{2}^{3 n-1} e^{n t_{1}} & \mathcal{F}_{112}=\sum_{n} \frac{n^{2} N_{n}}{(3 n-2) !} t_{2}^{3 n-2} e^{n t_{1}} \\
\mathcal{F}_{122}=\sum_{n} \frac{n N_{n}}{(3 n-3) !} t_{2}^{3 n-3} e^{n t_{1}} & \mathcal{F}_{222}=\sum_{n} \frac{N_{n}}{(3 n-4) !} t_{2}^{3 n-4} e^{n t_{1}}
\end{array}
$$

One can easily check that every equation in 152 is true if and only if

$$
\mathcal{F}_{222}=\mathcal{F}_{112}^{2}-\mathcal{F}_{111} \mathcal{F}_{122} .
$$


Indeed,

$$
\begin{aligned}
& \mathcal{F}_{1} \mathcal{F}_{0}^{-1} \mathcal{F}_{2}=\left(\begin{array}{ccc}
0 & \mathcal{F}_{112} & \mathcal{F}_{122} \\
\mathcal{F}_{112} & \mathcal{F}_{122}+\mathcal{F}_{111} \mathcal{F}_{112} & \mathcal{F}_{222}+\mathcal{F}_{111} \mathcal{F}_{122} \\
\mathcal{F}_{122} & \mathcal{F}_{112}^{2} & \mathcal{F}_{112} \mathcal{F}_{122}
\end{array}\right) \\
& \mathcal{F}_{0} \mathcal{F}_{1}^{-1} \mathcal{F}_{2}=\frac{1}{\mathcal{F}_{122}}\left(\begin{array}{ccc}
-\mathcal{F}_{112} & \mathcal{F}_{122} & \mathcal{F}_{222} \\
\mathcal{F}_{122} & 0 & 0 \\
\mathcal{F}_{112}^{2}-\mathcal{F}_{111} \mathcal{F}_{122} & 0 & \mathcal{F}_{122}^{2}-\mathcal{F}_{112} \mathcal{F}_{222}
\end{array}\right) \\
& \mathcal{F}_{0} \mathcal{F}_{2}^{-1} \mathcal{F}_{1}=\frac{1}{\mathcal{F}_{112} \mathcal{F}_{222}-\mathcal{F}_{122}^{2}}\left(\begin{array}{ccc}
-\mathcal{F}_{122} & \mathcal{F}_{112}^{2}-\mathcal{F}_{111} \mathcal{F}_{122} & 0 \\
\mathcal{F}_{222} & \mathcal{F}_{111} \mathcal{F}_{222}-\mathcal{F}_{112} \mathcal{F}_{122} & \mathcal{F}_{112} \mathcal{F}_{222}-\mathcal{F}_{122}^{2} \\
0 & \mathcal{F}_{112} \mathcal{F}_{222}-\mathcal{F}_{122}^{2} & 0
\end{array}\right)
\end{aligned}
$$

Eq. 180 is the famous equation, providing the recursive relations for $N_{n}$ 49:

$$
\frac{N_{n}}{(3 n-4) !}=\sum_{a+b=n} \frac{a^{2} b(3 b-1) b(2 a-b)}{(3 a-1) !(3 b-1) !} N_{a} N_{b} .
$$

For example, $N_{2}=N_{1}^{2}, N_{3}=12 N_{1} N_{2}=12 N_{1}^{3}, \ldots$

The zero curvature condition (159) is obviously satisfied for $\eta^{0}=\mathcal{F}_{0}: R_{i j k l}\left(\eta^{(0)}\right)=0$, but it is not fulfilled already for $\eta^{(1)}=\mathcal{F}_{1}$ :

$$
R_{1212}\left(\eta^{(1)}\right) \sim \mathcal{F}_{1112} \mathcal{F}_{1222}-\mathcal{F}_{1122}^{2}=-N_{1}^{2} e^{3 t_{1}}+\ldots \neq 0 .
$$

Now, let us turn to the leading (perturbative) approximation to the exact Seiberg-Witten prepotential, which satisfies (152) by itself. The perturbative contribution is non-transcendental, thus calculation can be performed in explicit form:

$$
\begin{aligned}
\mathcal{F}_{\text {pert }} \equiv & \mathcal{F}\left(a_{i}\right)=\left.\frac{1}{2} \sum_{\substack{m<n \\
m, n=1}}^{N}\left(A_{m}-A_{n}\right)^{2} \log \left(A_{m}-A_{n}\right)\right|_{\sum_{m} A_{m}=0}= \\
& =\frac{1}{2} \sum_{\substack{i<j \\
i, j=1}}^{N-1}\left(a_{i}-a_{j}\right)^{2} \log \left(a_{i}-a_{j}\right)+\frac{1}{2} \sum_{i=1}^{N-1} a_{i}^{2} \log a_{i}
\end{aligned}
$$

Here we took $a_{i}=A_{i}-A_{N}$ - one of the many possible choices of independent variables, which differ by linear transformations. According to (155) the system (152) is covariant under such changes. 
We shall use the notation $a_{i j}=a_{i}-a_{j}$. The matrix

$$
\begin{aligned}
& \left\{\left(\mathcal{F}_{1}\right)_{m n}\right\}=\left\{\frac{\partial^{3} \mathcal{F}}{\partial a_{1} \partial a_{m} \partial a_{n}}\right\}= \\
& =\left(\begin{array}{ccccc}
\frac{1}{a_{1}}+\sum_{l \neq 1} \frac{1}{a_{1 l}} & -\frac{1}{a_{12}} & -\frac{1}{a_{13}} & -\frac{1}{a_{14}} & \\
-\frac{1}{a_{12}} & +\frac{1}{a_{12}} & 0 & 0 & \\
-\frac{1}{a_{13}} & 0 & +\frac{1}{a_{13}} & 0 & \ldots \\
-\frac{1}{a_{14}} & 0 & 0 & +\frac{1}{a_{14}} & \\
& & \ldots &
\end{array}\right)
\end{aligned}
$$

i.e.,

$$
\begin{gathered}
\left\{\left(\mathcal{F}_{i}\right)_{m n}\right\}=\frac{\delta_{m n}\left(1-\delta_{m i}\right)\left(1-\delta_{n i}\right)}{a_{i m}}-\frac{\delta_{m i}\left(1-\delta_{n i}\right)}{a_{i n}}-\frac{\delta_{n i}\left(1-\delta_{m i}\right)}{a_{i m}}+ \\
+\left(\frac{1}{a_{i}}+\sum_{l \neq i} \frac{1}{a_{i k}}\right) \delta_{m i} \delta_{n i}
\end{gathered}
$$

The inverse matrix

$$
\left\{\left(\mathcal{F}_{k}^{-1}\right)_{m n}\right\}=a_{k}+\delta_{m n} a_{k m}\left(1-\delta_{m k}\right)
$$

for example

$$
\left\{\left(\mathcal{F}_{1}^{-1}\right)_{m n}\right\}=a_{1}\left(\begin{array}{cccc}
1 & 1 & 1 & \cdot \\
1 & 1 & 1 & \cdot \\
1 & 1 & 1 & \cdot \\
& \ldots & &
\end{array}\right)+\left(\begin{array}{cccc}
0 & 0 & 0 & \cdot \\
0 & a_{12} & 0 & \cdot \\
0 & 0 & a_{13} & \cdot \\
& \ldots & &
\end{array}\right)
$$

As the simplest example let us consider the case $N=4$. We already know that for $N=4$ it is sufficient to check only one of the eqs.(152), all the others follow automatically. We take $k=1$. Then,

$$
\begin{aligned}
& \mathcal{F}_{1}=\left(\begin{array}{ccc}
\frac{1}{a_{1}}+\frac{1}{a_{12}}+\frac{1}{a_{13}} & -\frac{1}{a_{12}} & -\frac{1}{a_{13}} \\
-\frac{1}{a_{12}} & \frac{1}{a_{12}} & 0 \\
-\frac{1}{a_{13}} & 0 & \frac{1}{a_{13}}
\end{array}\right) \quad \mathcal{F}_{2}^{-1}=\left(\begin{array}{ccc}
a_{2}+a_{21} & a_{2} & a_{2} \\
a_{2} & a_{2} & a_{2} \\
a_{2} & a_{2} & a_{2}+a_{23}
\end{array}\right) \\
& \mathcal{F}_{3}=\left(\begin{array}{ccc}
\frac{1}{a_{31}} & 0 & -\frac{1}{a_{31}} \\
0 & \frac{1}{a_{32}} & -\frac{1}{a_{32}} \\
-\frac{1}{a_{31}} & -\frac{1}{a_{32}} & \frac{1}{a_{3}}+\frac{1}{a_{31}}+\frac{1}{a_{32}}
\end{array}\right)
\end{aligned}
$$

and, say,

$$
\mathcal{F}_{1} \mathcal{F}_{2}^{-1} \mathcal{F}_{3}=\left(\begin{array}{ccc}
\star & -\frac{1}{a_{31}} & \Delta+\frac{a_{21}+a_{23}}{a_{13}^{2}} \\
-\frac{1}{a_{13}} & \star & \frac{1}{a_{13}} \\
\frac{a_{21}+a_{23}}{a_{13}^{2}} & \frac{1}{a_{13}} & \star
\end{array}\right)
$$


where we do not write down manifestly the diagonal terms since, to check (152), one only needs to prove the symmetricity of the matrix. This is really the case, since

$$
\Delta \equiv \frac{a_{2}}{a_{1} a_{3}}-\frac{a_{21}}{a_{1} a_{31}}-\frac{a_{23}}{a_{3} a_{13}}=0
$$

Only at this stage we use manifestly that $a_{i j}=a_{i}-a_{j}$.

Now let us prove (152) for the general case. We check the equation for the inverse matrices. Namely, using formulas (186)-(187), one obtains

$$
\begin{gathered}
\left(\mathcal{F}_{i}^{-1} \mathcal{F}_{j} \mathcal{F}_{k}^{-1}\right)_{\alpha \beta}= \\
=\frac{a_{i} a_{k}}{a_{j}}+\delta_{\alpha \beta}\left(1-\delta_{i \alpha}\right)\left(1-\delta_{k \alpha}\right)\left(1-\delta_{j \alpha}\right) \frac{a_{i \alpha} a_{k \beta}}{a_{j \beta}}+\delta_{j \alpha} \delta_{j \beta}\left(1-\delta_{i \alpha}\right)\left(1-\delta_{k \beta}\right)\left(\frac{1}{a_{j}}+\sum_{n \neq j} \frac{1}{a_{j n}}\right)+ \\
+\delta_{j \alpha}\left(1-\delta_{i \alpha}\right) a_{i \alpha}\left(\frac{a_{k}}{a_{j}}-\frac{a_{k \beta}}{a_{j \beta}}\left(1-\delta_{k \beta}\right)\left(1-\delta_{j \beta}\right)\right)+\delta_{j \beta}\left(1-\delta_{k \beta}\right)\left(\frac{a_{i}}{a_{j}}-\frac{a_{i \alpha}}{a_{j \alpha}}\left(1-\delta_{i \alpha}\right)\left(1-\delta_{j \alpha}\right)\right)= \\
=\frac{a_{i} a_{k}}{a_{j}}+\delta_{\alpha \beta}\left(1-\delta_{i \alpha}-\delta_{k \alpha}-\delta_{j \alpha}\right) \frac{a_{i \alpha} a_{k \beta}}{a_{j \beta}}+\delta_{j \alpha} \delta_{j \beta}\left(\frac{1}{a_{j}}+\sum_{n \neq j} \frac{1}{a_{j n}}\right)+ \\
+\delta_{j \alpha} a_{i \alpha}\left(\frac{a_{k}}{a_{j}}-\frac{a_{k \beta}}{a_{j \beta}}\left(1-\delta_{k \beta}-\delta_{j \beta}\right)\right)+\delta_{j \beta}\left(\frac{a_{i}}{a_{j}}-\frac{a_{i \alpha}}{a_{j \alpha}}\left(1-\delta_{i \alpha}-\delta_{j \alpha}\right)\right)
\end{gathered}
$$

where we used that $i \neq j \neq k$. The first three terms are evidently symmetric with respect to interchanging

$\alpha \leftrightarrow \beta$. In order to prove the symmetricity of the last two terms, we need to use the identities $\frac{a_{k}}{a_{j}}-\frac{a_{k \beta}}{a_{j \beta}}=$ $\frac{a_{\beta} a_{j k}}{a_{j} a_{j \beta}} \stackrel{k=\beta}{\rightarrow} \frac{a_{k}}{a_{j}}, \frac{a_{i}}{a_{j}}-\frac{a_{i \alpha}}{a_{j \alpha}}=\frac{a_{\alpha} a_{j i}}{a_{j} a_{j \alpha}} \stackrel{i=\alpha}{\rightarrow} \frac{a_{i}}{a_{j}}$. Then, one gets

the last line of $(192)=\delta_{j \alpha}\left(1-\delta_{j \beta}\right) \frac{a_{i j} a_{j k}}{a_{j}} \frac{a_{\beta}}{a_{j \beta}}+\delta_{j \beta}\left(1-\delta_{j \alpha}\right) \frac{a_{i j} a_{j k}}{a_{j}} \frac{a_{\alpha}}{a_{j \alpha}}+\delta_{j \alpha} \delta_{j \beta} \frac{a_{k} a_{i \alpha}+a_{i} a_{k \beta}}{a_{j}}$

It is interesting to note that in the particular example (184), all the metrics $\eta^{(k)}$ are flat. Moreover, it is easy to find the explicit flat coordinates:

$$
\begin{gathered}
\eta^{(k)}=\eta_{i j}^{(k)} d a^{i} d a^{j}=\mathcal{F}_{i j k} d a^{i} d a_{j}=d a_{i} d a_{j} \partial_{i j}^{2}\left(\partial_{k} \mathcal{F}\right)= \\
=\frac{d a_{k}^{2}}{a_{k}}+\sum_{l \neq k} \frac{d a_{k l}^{2}}{a_{k l}}=4\left(\left(d \sqrt{a_{k}}\right)^{2}+\sum_{l \neq k}\left(d \sqrt{a_{k l}}\right)\right) .
\end{gathered}
$$

\section{Conclusion}

The picture presented above should be actually considered as a simple version of a generic nonperturbative effective target-space formulation of string theory. String theory possesses a huge amount of "hidden symmetries" which allow one sometimes to determine the answer without a direct computation. The introduced objects have a direct generalization for the whole string theory picture where at the moment only some observations based on consistency requirements for the relations among dual theories are made 12]. The difference of the presented above picture with generic conception of string duality is that the above construction is formulated in strict mathematical sense what still remains to be done for more "rich" string models.

A stringy generalization is straightforward and related first of all with the prepotentials arising in the study of realistic models related to the Calabi-Yau compactifications. All the steps described above can be in principle 
repeated leading finally to the integrable models based on the higher-dimensional complex manifolds (instead of $1_{\mathbf{C}}$-dimensional $\Sigma$ ). Such integrable systems are not investigated yet in detail (see however [31, 52]).

Another problem is that even for the simplest cases considered above the complete picture still has a lot of open questions. In particular the exact form of the full generating function $\log \mathcal{T}$ is not yet known even for the Seiberg-Witten effective theories. One can also notice that the explicit examples presented above were usually restricted to the case of $S U\left(N_{c}\right)$ gauge groups and only their simplest representations, in principle there should exist an invariant language applicable to any gauge group and any representation.

One more direction is related with the study of effective theories on (partially) compactified target-spaces [53, 54]. The compactification of one dimension leads to appearance of the well-known class of relativistic integrable models [55] and allows one to interpret the divisor on a complex curve corresponding to a finite-gap solution in terms of the loop variables. This question, however, deserves further investigation.

In spite of all the problems it is easy to believe that for all the theories where it is possible to make any statement about the nonperturbative and exact quantities there exists something more than a summation of a perturbation theory. The main idea I tried to advocate above that this could be the principle of integrability, which has been checked already in several examples and based on general belief that the realistic theory should be a selfconsistent one and adjust automatically its properties not to be ill-defined both at large and small distances. It looks that an adequate language for the effective formulation of nonperturbative field and string theories obeying such property can be looked for among integrable systems.

\section{$7 \quad$ Acknowledgments}

I am deeply indebted to A.Gorsky, S.Kharchev, I.Krichever, A.Mironov and A.Morozov for the fruitful collaboration on various questions discussed above in these notes and many illuminating discussions. I am grateful to B.Dubrovin, V.Fock, A.Gerasimov, P.Grinevich, S.Gukov, A.Gurevich, I.Kolokolov, A.Losev, Yu.Manin, N.Nekrasov, A.Rosly, A.Orlov, V.Rubtsov and A.Zabrodin for many discussions and explanations and I am also grateful to M.Bianchi, P.Fre, D.Lüst, A.Sagnotti, J.Schwarz, I.Tyutin, B.Voronov and P.West for interesting discussions concerning relation of the topic of this paper to more conventional problems of string and field theories. The work was in part supported by RFFI grant 96-01-00887 and INTAS grant INTAS-93-2058. I am grateful to M.Martellini for warm hospitality in Como where this work was completed.

\section{References}

[1] V.Kazakov, Mod.Phys.Lett. A4 (1989) 2125;

E.Brézin and V.Kazakov, Phys.Lett. B236 (1990) 144;

M.Douglas and S.Shenker, Nucl.Phys. B335 (1990) 635;

D.Gross and A.Migdal, Phys.Rev.Lett. 64 (1990) 127.

[2] M.Fukuma, H.Kawai and R.Nakayama, Int.J.Mod.Phys. A6 (1991) 1385;

R.Dijkgraaf, H.Verlinde and E.Verlinde, Nucl.Phys. B348 (1991) 435. 
[3] M.Kontsevich, Func.Anal.\& Apps. 25 (1991) 50; Comm. Math.Phys. 147 (1992) 1.

[4] S.Kharchev, A.Marshakov, A.Mironov, A.Morozov and A.Zabrodin, Nucl.Phys. B380 (1992) 181.

[5] S.Kharchev, A.Marshakov, A.Mironov and A.Morozov., Mod.Phys.Lett. A8 (1993) 1047.

[6] N.Seiberg and E.Witten, Nucl.Phys. B426 (1994) 19; hepth/9407087.

[7] N.Seiberg and E.Witten, Nucl.Phys. B431 (1994) 484; hepth/9408099.

[8] C.Vafa and E.Witten, hepth/9408074.

[9] A.Gorsky, I.Krichever, A.Marshakov, A.Mironov and A.Morozov, Phys.Lett. B355 (1995) 466; hepth/9505035.

[10] L.Faddeev and L.Takhtadjan, Hamiltonian Approach to the Theory of Solitons, 1986.

[11] F.Smirnov, Form Factors in Completely Integrable Models of Quantum Field Theory, Adv.Series in Math.Phys. 14 (1992), World Scientific PC;

A.Its, A.Izergin, V.Korepin and N.Slavnov, Phys.Rev.Lett. 70 (1993) 1704 and references therein;

M.Jimbo and T.Miwa, Algebraic Analysis of Solvable Lattice Models, Conf.Board of the Math.Sci., Regional Conf.Series in Math. 85 (1995);

S.Khoroshkin, D.Lebedev and S.Pakuliak, q-alg/9602030 and references therein.

[12] J.Schwarz, Lectures on superstring and $M$ theory dualities, hepth/9607201 and references therein.

[13] S.Kachru and C.Vafa, hepth/9505105;

S.Ferrara, J.Harvey, A.Strominger and C.Vafa, hepth/9505162;

A.Klemm, W.Lerche, P.Mayr, C.Vafa and N.Warner, hepth/9604034.

[14] R.Donagi and E.Witten, hepth/9510101.

[15] E.Martinec, hepth/9510204.

[16] A.Gorsky and A.Marshakov, hepth/9510224, Phys.Lett. B375 (1996) 127.

[17] E.Martinec and N.Warner, hepth/9511052.

[18] H.Itoyama and A.Morozov, hepth/9511126;hepth/9512161;hepth/9601168.

[19] A.Marshakov, Mod.Phys.Lett. A11 (1996) 1169; hepth/9602005.

[20] C.Ann and S.Nam, hepth/9603028.

[21] A.Klemm, W.Lerche, S.Theisen and S.Yankielowicz, Phys.Lett. 344B (1995) 169; hepth/9411048;

P.Argyres and A.Faraggi, Phys.Rev.Lett. 73 (1995) 3931, hepth/9411057.

[22] A.Hanany and Y.Oz, hepth/9505075;

P.Argyres, D.Plesser and A.Shapere, hepth/9505100;

J.Minahan and D.Nemeschansky, hepth/9507032;

P.Argyres and A.Shapere, hepth/9509175;

A.Hanany, hepth/9509176. 
[23] A.Gorsky, A.Marshakov, A.Mironov and A.Morozov, hepth/9603140, Phys.Lett. B380 (1996) 75;

A.Gorsky, A.Marshakov, A.Mironov and A.Morozov, hepth/9604078, in Problems in Modern Theoretical Physics, Dubna 1996, 44-62.

[24] B.Dubrovin, I.Krichever and S.Novikov, Integrable systems - I, Sovremennye problemy matematiki (VINITI), Dynamical systems - 4 (1985) 179.

[25] I.Krichever and D.Phong, hepth/9604199.

[26] A.Marshakov, From Nonperturbative Supersymmetric Quantum Gauge Theories to Integrable Systems, preprint FIAN/TD-11/96, ITEP/TH-23/96, hepth/9607159.

[27] E.Witten, Surv.Diff.Geom. 1 (1991) 243;

R.Dijkgraaf, E.Verlinde and H.Verlinde, Nucl.Phys. B352 (1991) 59.

[28] I.Krichever, On Heisenberg relations for the ordinary linear differential operators, preprint ETH (1990);

S.Novikov, Func.Anal.\& Appl. 24 (1990) 43;

G.Moore, Comm.Math.Phys. 133(1990) 261;

R.Schimmrigk, Phys.Rev.Lett. 65 (1990) 2483.

[29] I.Krichever, Comm.Pure Appl.Math. 47 (1994) 437; Preprint LPTENS-92-18.

[30] B.Dubrovin, hepth/9407018; Nucl.Phys. B379 (1992) 627.

[31] N. Hitchin, Duke.Math.Journ. 54 (1987) 91.

[32] I.Krichever, in the Appendix to B.Dubrovin, Uspekhi Mat.Nauk, 36 (1981) N2, 12.

[33] E.Sklyanin, J.Sov.Math. 47 (1989) 2473.

[34] I.Krichever, Func.Anal. \& Appl.14 (1980) 282.

[35] E.Sklyanin, Alg.Anal. 6 (1994) 227;

B.Enriquez and V.Rubtsov, alg-geom/9503010;

N.Nekrasov, hepth/9503133;

G.Arutyunov and P.Medvedev, hepth/9511070.

[36] V.Inozemtsev, Comm.Math.Phys. 121 (1989) 629.

[37] L.Lipatov, JETP.Lett. 59 (1994) 596.

[38] L.Faddeev and G.Korchemsky, Phys.Lett. B342 (1994) 311;

G.Korchemsky, Nucl.Phys. B443 (1995) 255.

[39] E.Sklyanin, Func.Anal \& Apps. 16 (1982) 27; 17 (1983) 34.

[40] S.Kharchev and A.Marshakov, Int.J.Mod.Phys. A10 (1995) 1219.

[41] I.Krichever, Uspekhi Mat. Nauk, 44 (1989), N2, 121. 
[42] V.Fock, A.Gorsky, N.Nekrasov and V.Rubtsov, Preprint ITEP/TH-36/96;

V.Fock, Three remarks on group invariants related to flat connections, Preprint ITEP/TH-5/96.

[43] I.Krichever, Func.Anal.\& Apps. 22 (1988) 37; Comm.Math.Phys. 143 (1991) 415

[44] B.Dubrovin and S.Novikov, Uspekhi Mat.Nauk, 44 (1989) N6, 29.

[45] A.Gurevich and L.Pitaevsky, JETP, 65 (1973)65; see also S.Novikov, S.Manakov, L.Pitaevsky and V.Zakharov "Theory of solitons", Moscow 1980.

[46] E.Martinec and N.Warner, hepth/9509161.

[47] T.Nakatsu and K.Takasaki, hepth/9509162.

[48] Yu.Manin, Frobenius manifolds, quantum cohomology and moduli spaces, Preprint MPI, 1996.

[49] M.Kontsevich and Yu.Manin, Comm.Math.Phys. 164 (1994) 525.

[50] A.Marshakov, A.Mironov and A.Morozov, WDVV-like equations in N=2 SUSY Yang-Mills Theory, preprint FIAN/TD-10/96, ITEP/TH-22/96, hepth/9607109, to appear in Phys.Lett. B.

[51] A.Marshakov, A.Mironov and A.Morozov, to appear.

[52] E.Markman, Comp.Math. 93 (1994) 255;

R.Donagi and E.Markman, Cubics, integrable systems and Calabi-Yau Threefolds, preprint; Spectral covers, algebraically completely integrable Hamiltonian systems and moduli of bundles, preprint.

[53] N.Seiberg and E.Witten, hepth/9609219.

[54] N.Nekrasov, hepth/9609219.

[55] S.Ruijsenaars, Finite-dimensional Soliton Systems, in Integrable and Super-Integrable Systems, World Scientific, 1989. 